\title{
Catalytic upgrading of pyrolysis wax-oil obtained from waxed corrugated cardboard using zeolite $Y$ catalyst
}

Farid Sotoudehnia, Berlinda Orji, Endalkachew Mengistie, Abdulbaset M. Alayat, Armando G. McDonald*

Department of Forest, Rangeland and Fire Sciences, University of Idaho, Moscow, Idaho, USA *corresponding author, armandm@uidaho.edu

\section{Supplemental Material}

Table S1. Identified compounds in gasoline and the liquid products of catalytic pyrolysis of solvent extracted wax and wax-oil samples collected from the U-tube. The units are mg compound per g resultant liquid product.

\begin{tabular}{|c|c|c|c|c|c|c|c|c|}
\hline Compound Name & $\mathbf{M +}$ & Formula & $\begin{array}{l}\text { RT } \\
\min \end{array}$ & $\begin{array}{c}\text { Gasoline } \\
\mathrm{mg} / \mathrm{g}\end{array}$ & $\begin{array}{c}\text { Ext Wax } \\
\text { mg/g }\end{array}$ & $\begin{array}{c}\text { WO450 } \\
\mathrm{mg} / \mathrm{g}\end{array}$ & $\begin{array}{c}\text { WO500 } \\
\text { mg/g }\end{array}$ & $\begin{array}{c}\text { WO550 } \\
\mathrm{mg} / \mathrm{g}\end{array}$ \\
\hline 2,2-Dimethylbutane & 86 & $\mathrm{C}_{6} \mathrm{H}_{14}$ & 1.99 & 19.71 & - & - & - & - \\
\hline Pentane, 3-methylene- & 84 & $\mathrm{C}_{6} \mathrm{H}_{12}$ & 2.1 & 29.60 & 17.93 & - & 2.14 & 2.89 \\
\hline 2-Pentene, 3-methyl-, (E)- & 84 & $\mathrm{C}_{6} \mathrm{H}_{12}$ & 2.15 & - & 11.00 & - & - & 3.05 \\
\hline Cyclopentane, methyl- & 84 & $\mathrm{C}_{6} \mathrm{H}_{12}$ & 2.35 & 32.38 & - & 6.26 & - & 2.19 \\
\hline Cyclopropane, 1,1,2,3-tetramethyl- & 98 & $\mathrm{C}_{7} \mathrm{H}_{14}$ & 2.62 & - & 25.08 & 44.09 & - & 0.44 \\
\hline Pentane, 2,4-dimethyl- & 100 & $\mathrm{C}_{7} \mathrm{H}_{16}$ & 2.71 & 73.92 & - & 19.70 & - & - \\
\hline Hexane, 2-methyl- & 100 & $\mathrm{C}_{7} \mathrm{H}_{16}$ & 2.76 & - & 50.68 & - & 2.92 & 12.05 \\
\hline Pentane, 3-ethyl- & 100 & $\mathrm{C}_{7} \mathrm{H}_{16}$ & 2.81 & 32.31 & 40.99 & 14.74 & 2.59 & 8.12 \\
\hline 2,2,3,3-Tetramethylbutane & 114 & $\mathrm{C}_{8} \mathrm{H}_{18}$ & 2.97 & 65.80 & 8.76 & - & 0.36 & 2.12 \\
\hline 1-Heptene, 4-methyl- & 112 & $\mathrm{C}_{8} \mathrm{H}_{16}$ & 3.01 & - & - & - & - & - \\
\hline Hexane, 3-methyl- & 100 & $\mathrm{C}_{7} \mathrm{H}_{16}$ & 3.13 & 25.18 & 10.97 & 5.47 & 0.95 & 1.77 \\
\hline 1,4-Pentadiene, 3,3-dimethyl- & 96 & $\mathrm{C}_{7} \mathrm{H}_{12}$ & 3.2 & 6.79 & - & - & - & - \\
\hline 4-Methyl-2-hexene,c\&t & 98 & $\mathrm{C}_{7} \mathrm{H}_{14}$ & 3.23 & - & 11.32 & 8.11 & 0.22 & 2.06 \\
\hline 2-Hexene, 3-methyl-, (Z)- & 98 & $\mathrm{C}_{7} \mathrm{H}_{14}$ & 3.33 & - & 2.55 & 2.32 & - & 0.37 \\
\hline 3-Heptene & 98 & $\mathrm{C}_{7} \mathrm{H}_{14}$ & 3.4 & - & 2.71 & 2.33 & - & 0.44 \\
\hline Cyclohexane, methyl- & 95 & $\mathrm{C}_{7} \mathrm{H}_{14}$ & 3.5 & 23.12 & 5.86 & 3.62 & 0.60 & 1.63 \\
\hline Hexane, 2,5-dimethyl- & 114 & $\mathrm{C}_{8} \mathrm{H}_{18}$ & 3.59 & 6.46 & - & - & - & - \\
\hline Hexane, 2,4-dimethyl- & 114 & $\mathrm{C}_{8} \mathrm{H}_{18}$ & 3.63 & 11.61 & 26.61 & 15.18 & 1.32 & 1.17 \\
\hline Cyclopentane, 1,2,4-trimethyl- & 112 & $\mathrm{C}_{8} \mathrm{H}_{18}$ & 3.77 & 4.72 & - & - & 2.60 & 3.45 \\
\hline
\end{tabular}




\begin{tabular}{|c|c|c|c|c|c|c|c|c|}
\hline 2-Pentene, 3-ethyl-2-methyl- & 112 & $\mathrm{C}_{8} \mathrm{H}_{16}$ & 3.8 & - & 2.20 & - & 0.34 & 0.58 \\
\hline 3-Ethylpentane & 100 & $\mathrm{C}_{7} \mathrm{H}_{16}$ & 3.92 & 19.68 & - & - & - & - \\
\hline 2,3,3-Trimethylpentane & 114 & $\mathrm{C}_{8} \mathrm{H}_{18}$ & 4.02 & 14.22 & 2.86 & 3.20 & - & 0.66 \\
\hline 2,3-Dimethylhexane & 114 & $\mathrm{C}_{8} \mathrm{H}_{18}$ & 4.11 & 9.73 & 11.37 & 6.00 & 2.05 & 1.57 \\
\hline 2-Methylheptane & 114 & $\mathrm{C}_{8} \mathrm{H}_{18}$ & 4.22 & 21.50 & 46.58 & 34.12 & 11.07 & 10.17 \\
\hline Toluene & 92 & $\mathrm{C}_{7} \mathrm{H}_{8}$ & 4.31 & 103.69 & 99.20 & 49.49 & - & 8.01 \\
\hline 3-Methylheptane & 114 & $\mathrm{C}_{8} \mathrm{H}_{18}$ & 4.37 & 21.92 & 43.04 & 33.90 & 4.58 & - \\
\hline Cyclohexane, 1,3-dimethyl-, trans- & 112 & $\mathrm{C}_{8} \mathrm{H}_{16}$ & 4.49 & 6.27 & 4.15 & 5.76 & 10.24 & 7.33 \\
\hline 2,2,4-Trimethylhexane & 128 & $\mathrm{C}_{9} \mathrm{H}_{20}$ & 4.55 & 7.25 & - & - & 0.70 & 0.99 \\
\hline Ethylcyclohexane & 112 & $\mathrm{C}_{8} \mathrm{H}_{16}$ & 4.69 & 3.27 & 3.36 & - & - & - \\
\hline 3-Ethylhexane & 114 & $\mathrm{C}_{8} \mathrm{H}_{18}$ & 4.93 & 17.64 & 12.77 & 12.49 & 3.18 & 2.03 \\
\hline 1,4-Dimethylcyclohexane & 112 & $\mathrm{C}_{8} \mathrm{H}_{16}$ & 5.07 & 4.13 & 2.18 & 5.32 & - & 0.74 \\
\hline Cyclohexane, 1,4-dimethyl-, cis- & 112 & $\mathrm{C}_{8} \mathrm{H}_{16}$ & 5.18 & 3.21 & 3.87 & 4.15 & 0.83 & 0.74 \\
\hline 2-Hexene, 3,5-dimethyl- & 112 & $\mathrm{C}_{8} \mathrm{H}_{18}$ & 5.21 & - & 3.25 & - & 0.54 & 0.59 \\
\hline 2,3-Dimethylheptane & 128 & $\mathrm{C}_{9} \mathrm{H}_{20}$ & 5.28 & 1.85 & - & 2.13 & 0.60 & 0.41 \\
\hline Heptane, 2,4-dimethyl- & 128 & $\mathrm{C}_{9} \mathrm{H}_{20}$ & 5.45 & 3.39 & 7.98 & 6.29 & 2.70 & 1.45 \\
\hline 2-Methyloctane & 128 & $\mathrm{C}_{9} \mathrm{H}_{20}$ & 5.6 & 4.00 & 8.80 & 7.83 & 3.10 & 1.49 \\
\hline 3,5-Dimethylheptane & 128 & $\mathrm{C}_{9} \mathrm{H}_{20}$ & 5.76 & 9.34 & 23.41 & 19.49 & 8.87 & 4.06 \\
\hline 1,2,4-Trimethylcyclohexane & 126 & $\mathrm{C}_{9} \mathrm{H}_{18}$ & 6.18 & 1.68 & 3.16 & 4.57 & 0.27 & 0.56 \\
\hline 3-Ethyl-2,4-dimethylpentane & 128 & $\mathrm{C}_{9} \mathrm{H}_{20}$ & 6.3 & 2.66 & 6.03 & 4.23 & 0.72 & 0.81 \\
\hline Ethylbenzene & 106 & $\mathrm{C}_{8} \mathrm{H}_{10}$ & 6.49 & 48.49 & 47.68 & - & 1.67 & 0.20 \\
\hline Octane, 2-methyl- & 128 & $\mathrm{C}_{9} \mathrm{H}_{20}$ & 6.53 & - & - & 49.87 & 0.42 & 10.04 \\
\hline p-Xylene & 106 & $\mathrm{C}_{8} \mathrm{H}_{10}$ & 6.71 & 129.08 & 74.27 & 118.67 & 20.30 & 20.60 \\
\hline 2,2,3-Trimethyldecane & 184 & $\mathrm{C}_{13} \mathrm{H}_{28}$ & 6.86 & 3.11 & - & - & - & - \\
\hline 2,2-Dimethyloctane & 142 & $\mathrm{C}_{10} \mathrm{H}_{22}$ & 7.05 & 4.00 & - & - & - & 0.26 \\
\hline Cyclohexane, 1-ethyl-2-methyl-, cis- & 126 & $\mathrm{C}_{9} \mathrm{H}_{18}$ & 7.14 & 2.54 & - & - & - & - \\
\hline o-Xylene & 106 & $\mathrm{C}_{8} \mathrm{H}_{10}$ & 7.34 & 49.38 & 20.08 & 40.63 & 35.29 & 5.71 \\
\hline 1-Nonene & 126 & $\mathrm{C}_{9} \mathrm{H}_{18}$ & 7.47 & 9.35 & 12.20 & 13.15 & 8.64 & 1.59 \\
\hline Cyclohexane, 1-ethyl-2-methyl- & 128 & $\mathrm{C}_{9} \mathrm{H}_{20}$ & 7.67 & 2.19 & - & 5.54 & - & 0.53 \\
\hline Nonane & 128 & $\mathrm{C}_{9} \mathrm{H}_{20}$ & 7.73 & - & 3.51 & - & 3.77 & - \\
\hline 2,3-Dimethyloctane & 142 & $\mathrm{C}_{10} \mathrm{H}_{22}$ & 7.91 & 1.70 & 2.78 & - & 1.25 & 0.69 \\
\hline 2,4,6-Trimethylheptane & 142 & $\mathrm{C}_{10} \mathrm{H}_{22}$ & 7.96 & 1.33 & 6.65 & 3.66 & 1.93 & 0.65 \\
\hline 4-Methylnonane & 142 & $\mathrm{C}_{10} \mathrm{H}_{22}$ & 8.18 & 2.14 & - & 8.92 & - & - \\
\hline Cumene & 120 & $\mathrm{C}_{9} \mathrm{H}_{12}$ & 8.22 & 3.41 & 12.90 & - & 5.08 & 1.38 \\
\hline Octane, 2,3-dimethyl- & 142 & $\mathrm{C}_{10} \mathrm{H}_{22}$ & 8.34 & - & 8.36 & 6.82 & 2.97 & 1.04 \\
\hline 2,6-Dimethyloctane & 142 & $\mathrm{C}_{10} \mathrm{H}_{22}$ & 8.43 & 3.73 & 9.87 & 8.65 & 3.83 & 1.40 \\
\hline Heptane, 3,4,5-trimethyl- & 142 & $\mathrm{C}_{10} \mathrm{H}_{22}$ & 8.6 & 1.63 & 8.96 & - & - & - \\
\hline 3-Ethyl-2-methylheptane & 142 & $\mathrm{C}_{10} \mathrm{H}_{22}$ & 8.65 & 0.91 & - & 9.01 & 3.13 & 1.10 \\
\hline Propylbenzene & 120 & $\mathrm{C}_{9} \mathrm{H}_{12}$ & 9.07 & 15.00 & - & - & - & - \\
\hline Nonane, 4-methyl- & 142 & $\mathrm{C}_{10} \mathrm{H}_{22}$ & 9.11 & - & 10.12 & 11.33 & 4.00 & 1.53 \\
\hline
\end{tabular}




\begin{tabular}{|c|c|c|c|c|c|c|c|c|}
\hline 2,2,6-Trimethyloctane & 156 & $\mathrm{C}_{11} \mathrm{H}_{24}$ & 9.21 & 9.50 & 6.63 & 5.61 & 2.07 & 0.91 \\
\hline Benzene, 1-ethyl-3-methyl- & 120 & $\mathrm{C}_{9} \mathrm{H}_{12}$ & 9.3 & 64.52 & 12.20 & 8.33 & 4.39 & 1.11 \\
\hline Nonane, 2-methyl- & 142 & $\mathrm{C}_{10} \mathrm{H}_{22}$ & 9.37 & - & 32.03 & 45.72 & 14.71 & 6.33 \\
\hline Benzene, 1-ethyl-4-methyl- & 120 & $\mathrm{C}_{9} \mathrm{H}_{12}$ & 9.5 & 26.55 & 2.63 & 35.49 & 0.65 & 0.32 \\
\hline Benzene, 1,2,4-trimethyl- & 120 & $\mathrm{C}_{9} \mathrm{H}_{12}$ & 9.56 & - & 31.85 & - & 13.84 & 5.73 \\
\hline Benzene, 1-ethyl-2-methyl- & 120 & $\mathrm{C}_{9} \mathrm{H}_{12}$ & 9.84 & 16.27 & 3.44 & 6.83 & 1.60 & 0.79 \\
\hline 5-Decene, $(\mathrm{E})-$ & 140 & $\mathrm{C}_{10} \mathrm{H}_{20}$ & 10.02 & - & 1.86 & 2.59 & 18.57 & 0.77 \\
\hline Mesitylene & 120 & $\mathrm{C}_{9} \mathrm{H}_{12}$ & 10.25 & 63.98 & 36.47 & 56.14 & 2.29 & 8.33 \\
\hline Decane & 142 & $\mathrm{C}_{10} \mathrm{H}_{22}$ & 10.39 & 6.14 & 7.31 & 5.83 & 0.79 & 1.01 \\
\hline cis-3-Decene & 140 & $\mathrm{C}_{10} \mathrm{H}_{20}$ & 10.59 & - & - & 4.94 & 1.91 & 0.28 \\
\hline Isobutylbenzene & 134 & $\mathrm{C}_{10} \mathrm{H}_{14}$ & 10.65 & 1.25 & - & - & - & - \\
\hline Sec-butylbenzene & 134 & $\mathrm{C}_{10} \mathrm{H}_{14}$ & 10.75 & 1.84 & - & - & - & - \\
\hline Octane, 5-ethyl-2-methyl- & 156 & $\mathrm{C}_{11} \mathrm{H}_{24}$ & 10.84 & - & 5.61 & 4.63 & 3.03 & 0.61 \\
\hline Nonane, 2,5-dimethyl- & 156 & $\mathrm{C}_{11} \mathrm{H}_{24}$ & 11.03 & - & 5.16 & 2.81 & 2.73 & 0.62 \\
\hline 1,2,4-Trimethylbenzene & 120 & $\mathrm{C}_{9} \mathrm{H}_{12}$ & 11.11 & 17.86 & 4.02 & 10.10 & 2.26 & 1.74 \\
\hline Decane, 3,7-dimethyl- & 170 & $\mathrm{C}_{12} \mathrm{H}_{26}$ & 11.27 & - & 6.55 & 4.68 & 1.48 & 0.88 \\
\hline Allylbenzene & 118 & $\mathrm{C}_{9} \mathrm{H}_{10}$ & 11.42 & 2.02 & 8.70 & 3.38 & 1.20 & 0.61 \\
\hline Indane & 118 & $\mathrm{C}_{9} \mathrm{H}_{10}$ & 11.52 & 7.15 & 6.98 & 5.49 & 2.15 & 0.69 \\
\hline 1,3-Diethylbenzene & 134 & $\mathrm{C}_{10} \mathrm{H}_{14}$ & 11.92 & 3.75 & 4.76 & - & 3.20 & 0.18 \\
\hline 1-Methyl-3-propylbenzene & 134 & $\mathrm{C}_{10} \mathrm{H}_{14}$ & 11.97 & 12.38 & - & 10.45 & 5.49 & 0.56 \\
\hline $\begin{array}{l}\text { Bicyclo[3.1.0]hex-2-ene, 4-methylene- } \\
\text { 1-(1-methylethyl)- }\end{array}$ & 134 & $\mathrm{C}_{10} \mathrm{H}_{14}$ & 12.11 & 9.98 & 9.63 & 8.51 & 3.25 & 0.94 \\
\hline 2-Ethyl-p-xylene & 134 & $\mathrm{C}_{10} \mathrm{H}_{14}$ & 12.19 & 12.22 & 8.47 & 12.05 & 3.14 & 1.98 \\
\hline 2-Methyldecane & 156 & $\mathrm{C}_{11} \mathrm{H}_{24}$ & 12.3 & 4.04 & 13.92 & 5.33 & 2.32 & 2.19 \\
\hline 1-Methyl-2-propylbenzene & 134 & $\mathrm{C}_{10} \mathrm{H}_{14}$ & 12.44 & 3.05 & 10.26 & 1.07 & - & 1.10 \\
\hline 3-Methyldecane & 156 & $\mathrm{C}_{11} \mathrm{H}_{24}$ & 12.5 & 1.90 & 9.76 & 5.06 & 1.42 & 1.22 \\
\hline 1-Ethyl-2,4-dimethylbenzene & 134 & $\mathrm{C}_{10} \mathrm{H}_{14}$ & 12.75 & 6.58 & - & 6.93 & - & 1.81 \\
\hline Benzene, 4-ethyl-1,2-dimethyl- & 134 & $\mathrm{C}_{10} \mathrm{H}_{14}$ & 12.8 & 5.60 & 5.51 & 4.11 & 2.92 & 0.56 \\
\hline 4-Allyltoluene & 132 & $\mathrm{C}_{10} \mathrm{H}_{12}$ & 12.87 & 0.99 & 3.09 & - & 0.56 & - \\
\hline p-Cymene & 134 & $\mathrm{C}_{10} \mathrm{H}_{14}$ & 12.99 & 13.78 & 5.81 & 9.01 & 1.42 & 0.39 \\
\hline O-Cymene & 134 & $\mathrm{C}_{10} \mathrm{H}_{14}$ & 13.2 & 0.70 & 0.86 & 5.03 & 0.64 & 0.45 \\
\hline Undecane & 156 & $\mathrm{C}_{11} \mathrm{H}_{24}$ & 13.38 & 3.15 & 1.02 & 4.59 & - & - \\
\hline Benzene, 1-methyl-4-(2-methylpropyl)- & 148 & $\mathrm{C}_{11} \mathrm{H}_{16}$ & 13.46 & 0.86 & 5.30 & - & 0.46 & 0.80 \\
\hline Benzene, (1,1-dimethylpropyl)- & 148 & $\mathrm{C}_{11} \mathrm{H}_{16}$ & 13.55 & 0.91 & 0.88 & 2.64 & 0.31 & 0.07 \\
\hline 1-Ethyl-3,5-dimethylbenzene & 134 & $\mathrm{C}_{10} \mathrm{H}_{14}$ & 13.63 & 2.30 & 1.60 & 1.39 & 4.45 & 0.29 \\
\hline trans-4a-Methyl-decahydronaphthalene & 152 & $\mathrm{C}_{11} \mathrm{H}_{20}$ & 13.72 & 0.52 & 1.44 & - & - & 0.28 \\
\hline 1,2,3,4-Tetramethylbenzene, & 134 & $\mathrm{C}_{10} \mathrm{H}_{14}$ & 13.95 & - & 12.31 & 9.23 & 4.79 & 1.31 \\
\hline 1,2,4,5-Tetramethylbenzene & 134 & $\mathrm{C}_{10} \mathrm{H}_{14}$ & 14.04 & 9.34 & 12.02 & 10.30 & 1.39 & 1.51 \\
\hline 3,7-Dimethyldecane & 170 & $\mathrm{C}_{12} \mathrm{H}_{26}$ & 14.18 & 0.69 & 4.59 & 1.33 & 1.08 & 0.28 \\
\hline 1-Methyl-4-(1-methylpropyl)-benzene & 148 & $\mathrm{C}_{11} \mathrm{H}_{16}$ & 14.52 & 1.71 & 3.82 & 1.23 & 0.95 & 0.24 \\
\hline
\end{tabular}




\begin{tabular}{|c|c|c|c|c|c|c|c|c|}
\hline 5-Methylindan & 132 & $\mathrm{C}_{10} \mathrm{H}_{12}$ & 14.6 & 4.01 & 2.76 & 1.15 & 0.71 & 0.47 \\
\hline 1,3-Diethyl-4-methylbenzene & 148 & $\mathrm{C}_{11} \mathrm{H}_{16}$ & 14.71 & 1.52 & - & 2.73 & 0.52 & 0.14 \\
\hline 3,5-Diethyltoluene & 148 & $\mathrm{C}_{11} \mathrm{H}_{16}$ & 14.75 & 2.18 & 1.58 & 1.40 & 0.26 & 0.10 \\
\hline Benzene, 1-methyl-4-(1-methylpropyl)- & 148 & $\mathrm{C}_{11} \mathrm{H}_{16}$ & 14.88 & - & 1.20 & 0.99 & 3.70 & - \\
\hline 1-Methylindan & 132 & $\mathrm{C}_{10} \mathrm{H}_{12}$ & 14.93 & 5.06 & - & 2.68 & 1.52 & 0.38 \\
\hline 1,4-Diethylbenzene & 132 & $\mathrm{C}_{10} \mathrm{H}_{12}$ & 14.98 & 4.55 & 9.46 & 6.53 & 2.02 & 0.71 \\
\hline Isopentylbenzene & 148 & $\mathrm{C}_{11} \mathrm{H}_{16}$ & 15.12 & 2.20 & 4.33 & 2.97 & 1.69 & 0.50 \\
\hline $\begin{array}{l}\text { Benzene, 2,4-dimethyl-1-(1- } \\
\text { methylethyl)- }\end{array}$ & 148 & $\mathrm{C}_{11} \mathrm{H}_{16}$ & 15.28 & 3.08 & 5.91 & 3.42 & 0.63 & 0.52 \\
\hline 1,4-Diethyl-2-methylbenzene & 148 & $\mathrm{C}_{11} \mathrm{H}_{16}$ & 15.36 & 0.76 & - & - & - & - \\
\hline 3-Methylundecane & 170 & $\mathrm{C}_{12} \mathrm{H}_{26}$ & 15.45 & 0.98 & 4.76 & 2.78 & 0.33 & 0.80 \\
\hline Undecane, 3-methyl- & 170 & $\mathrm{C}_{12} \mathrm{H}_{26}$ & 15.68 & - & 1.49 & 1.30 & 0.63 & 0.25 \\
\hline $\begin{array}{l}\text { Benzene, 1-methyl-4-(1-methyl-2- } \\
\text { propenyl)- }\end{array}$ & 146 & $\mathrm{C}_{11} \mathrm{H}_{14}$ & 15.87 & 0.59 & - & - & - & - \\
\hline Naphthalene & 128 & $\mathrm{C}_{10} \mathrm{H}_{8}$ & 15.99 & 6.45 & 0.99 & 2.69 & 0.41 & 0.51 \\
\hline 1,1-Dimethylindan & 146 & $\mathrm{C}_{11} \mathrm{H}_{14}$ & 16 & 1.79 & 1.54 & 1.74 & 2.04 & 0.54 \\
\hline 2,2-Dimethylindene, 2,3-dihydro- & 146 & $\mathrm{C}_{11} \mathrm{H}_{14}$ & 16.07 & 0.95 & - & 1.65 & 0.62 & 0.98 \\
\hline Benzene, pentamethyl- & 148 & $\mathrm{C}_{11} \mathrm{H}_{16}$ & 16.3 & 3.68 & 6.28 & 4.14 & 1.98 & 0.20 \\
\hline Benzene, 1,4-diethyl-2-methyl- & 148 & $\mathrm{C}_{11} \mathrm{H}_{16}$ & 16.39 & - & - & 1.24 & 0.26 & 0.44 \\
\hline $\begin{array}{l}\text { Benzene, 1,3-dimethyl-5-(1- } \\
\text { methylethyl)- }\end{array}$ & 148 & $\mathrm{C}_{11} \mathrm{H}_{16}$ & 16.66 & - & - & 1.66 & 0.76 & 0.06 \\
\hline Dodecane & 170 & $\mathrm{C}_{12} \mathrm{H}_{26}$ & 16.73 & - & 4.91 & 1.56 & 0.90 & 0.12 \\
\hline Decane, 3,3,6-trimethyl- & 184 & $\mathrm{C}_{13} \mathrm{H}_{28}$ & 16.95 & - & 1.67 & 1.05 & 0.64 & 0.17 \\
\hline Undecane, 3,8-dimethyl- & 184 & $\mathrm{C}_{13} \mathrm{H}_{28}$ & 17.14 & - & 2.44 & 1.06 & 0.35 & 0.12 \\
\hline Undecane, 2,9-dimethyl- & 184 & $\mathrm{C}_{13} \mathrm{H}_{28}$ & 17.29 & - & 1.51 & 0.49 & 0.66 & 0.15 \\
\hline 4,7-Dimethylindan & 146 & $\mathrm{C}_{11} \mathrm{H}_{14}$ & 17.78 & 2.42 & - & - & 0.82 & 0.13 \\
\hline Dodecane, 6-methyl- & 184 & $\mathrm{C}_{13} \mathrm{H}_{28}$ & 17.81 & - & 1.98 & - & 0.69 & 0.26 \\
\hline Undecane, 2,4-dimethyl- & 184 & $\mathrm{C}_{13} \mathrm{H}_{28}$ & 17.85 & - & 2.14 & 3.36 & 0.70 & 0.26 \\
\hline Dodecane, 4-methyl- & 184 & $\mathrm{C}_{13} \mathrm{H}_{28}$ & 17.99 & - & 1.85 & 1.11 & 0.65 & 0.17 \\
\hline Dodecane, 2-methyl- & 184 & $\mathrm{C}_{13} \mathrm{H}_{28}$ & 18.12 & - & 2.24 & 1.44 & 0.70 & 0.31 \\
\hline 1,6-Dimethylindan & 146 & $\mathrm{C}_{11} \mathrm{H}_{14}$ & 18.17 & 1.04 & - & - & - & - \\
\hline Dodecane, 3-methyl- & 184 & $\mathrm{C}_{13} \mathrm{H}_{28}$ & 18.3 & - & 2.04 & 1.45 & 0.27 & 0.28 \\
\hline 3,4-Dimethylcumene & 148 & $\mathrm{C}_{11} \mathrm{H}_{16}$ & 18.68 & - & 1.79 & 2.72 & 1.39 & 0.19 \\
\hline Naphthalene, 2-methyl- & 142 & $\mathrm{C}_{11} \mathrm{H}_{10}$ & 19.05 & 6.92 & 3.87 & 7.02 & 0.86 & 1.07 \\
\hline Naphthalene, 1-methyl- & 142 & $\mathrm{C}_{11} \mathrm{H}_{10}$ & 19.51 & 2.94 & 2.52 & 3.68 & 0.25 & 0.16 \\
\hline Tridecane, 6-methyl- & 198 & $\mathrm{C}_{14} \mathrm{H}_{30}$ & 20.46 & - & 1.52 & 0.79 & 0.26 & 0.62 \\
\hline Tridecane, 5-methyl- & 198 & $\mathrm{C}_{14} \mathrm{H}_{30}$ & 20.54 & - & 1.09 & 0.34 & 0.27 & 0.08 \\
\hline Tridecane, 4-methyl- & 198 & $\mathrm{C}_{14} \mathrm{H}_{30}$ & 20.67 & - & 1.09 & 0.33 & 0.44 & 0.19 \\
\hline Tridecane, 2-methyl- & 198 & $\mathrm{C}_{14} \mathrm{H}_{30}$ & 20.8 & - & 1.40 & 0.74 & 0.29 & 0.13 \\
\hline Tridecane, 3-methyl- & 198 & $\mathrm{C}_{14} \mathrm{H}_{30}$ & 20.98 & - & 1.34 & 1.10 & 0.25 & 0.17 \\
\hline
\end{tabular}




\begin{tabular}{|l|c|c|c|c|c|c|c|c|}
\hline Naphthalene, 2-ethyl- & 156 & $\mathrm{C}_{12} \mathrm{H}_{12}$ & 21.71 & 0.68 & - & 1.32 & 0.36 & 0.36 \\
\hline Tetradecane & 198 & $\mathrm{C}_{14} \mathrm{H}_{30}$ & 21.75 & - & 1.71 & 3.79 & 1.01 & 0.40 \\
\hline Naphthalene, 2,6-dimethyl- & 156 & $\mathrm{C}_{12} \mathrm{H}_{12}$ & 21.97 & 0.84 & 2.75 & 2.89 & 0.57 & 0.53 \\
\hline Naphthalene, 1,7-dimethyl- & 156 & $\mathrm{C}_{12} \mathrm{H}_{12}$ & 22.35 & 0.91 & 1.57 & 1.72 & 0.36 & 0.36 \\
\hline Naphthalene, 1,5-dimethyl- & 156 & $\mathrm{C}_{12} \mathrm{H}_{12}$ & 22.44 & 0.45 & 1.01 & 1.12 & 0.19 & 0.21 \\
\hline Naphthalene, 1,3-dimethyl- & 156 & $\mathrm{C}_{12} \mathrm{H}_{12}$ & 22.99 & - & 0.94 & 0.75 & 0.26 & 0.09 \\
\hline Tetradecane, 4-methyl- & 212 & $\mathrm{C}_{15} \mathrm{H}_{32}$ & 23.21 & - & 0.56 & 0.47 & 0.19 & 0.14 \\
\hline Tetradecane, 2-methyl- & 212 & $\mathrm{C}_{15} \mathrm{H}_{32}$ & 23.34 & - & 0.87 & 0.87 & 0.26 & 0.08 \\
\hline Tetradecane, 3-methyl- & 212 & $\mathrm{C}_{15} \mathrm{H}_{32}$ & 23.52 & - & 0.64 & 0.50 & 0.19 & 0.12 \\
\hline Pentadecane & 212 & $\mathrm{C}_{15} \mathrm{H}_{32}$ & 24.24 & - & 0.81 & 0.45 & 0.24 & 0.08 \\
\hline Naphthalene, 2,3,6-trimethyl- & 170 & $\mathrm{C}_{13} \mathrm{H}_{14}$ & 25.02 & - & 0.80 & 0.97 & 0.34 & 0.29 \\
\hline Naphthalene, 1,4,5-trimethyl- & 170 & $\mathrm{C}_{13} \mathrm{H}_{14}$ & 25.13 & - & 0.82 & 1.10 & 0.24 & 0.16 \\
\hline Naphthalene, 1,6,7-trimethyl- & 170 & $\mathrm{C}_{13} \mathrm{H}_{14}$ & 25.52 & - & 0.78 & 1.05 & 0.32 & 0.10 \\
\hline Naphthalene, 1,4,6-trimethyl- & 170 & $\mathrm{C}_{13} \mathrm{H}_{14}$ & 25.92 & - & 0.99 & 1.39 & 0.24 & 0.12 \\
\hline
\end{tabular}

Table S2. Identified compounds in the liquid products of catalytic pyrolysis of solvent extracted wax and wax-oil samples collected from the impinger. The units are mg compound per $g$ resultant product.

\begin{tabular}{|c|c|c|c|c|c|c|c|}
\hline Compound Name & M+ & Formula & RT & $\begin{array}{c}\text { Ext Wax } \\
\text { mg/g }\end{array}$ & $\begin{array}{c}\text { WO450 } \\
\text { mg/g }\end{array}$ & $\begin{array}{c}\text { WO500 } \\
\text { mg/g }\end{array}$ & $\begin{array}{c}\text { WO } 550 \\
\text { mg/g }\end{array}$ \\
\hline Pentane, 3-methyl- & 86 & $\mathrm{C}_{6} \mathrm{H}_{14}$ & 2.05 & 103.48 & 54.84 & 96.39 & 108.26 \\
\hline 1-Hexene & 84 & $\mathrm{C}_{6} \mathrm{H}_{12}$ & 2.16 & 48.07 & 17.75 & 35.28 & 18.86 \\
\hline 2-Pentene, 3-methyl- & 84 & $\mathrm{C}_{6} \mathrm{H}_{12}$ & 2.21 & - & 29.01 & 14.78 & 20.86 \\
\hline Butane, 2,2,3-trimethyl- & 100 & $\mathrm{C}_{7} \mathrm{H}_{16}$ & 2.32 & 11.07 & 14.48 & 6.71 & 11.59 \\
\hline Pentane, 2,4-dimethyl- & 100 & $\mathrm{C}_{7} \mathrm{H}_{16}$ & 2.38 & 42.75 & 44.38 & 56.13 & 37.08 \\
\hline Cyclopropane, 1,1,2,3-tetramethyl- & 98 & $\mathrm{C}_{7} \mathrm{H}_{14}$ & 2.63 & 3.93 & 11.96 & 4.56 & 9.36 \\
\hline Hexane, 2-methyl- & 100 & $\mathrm{C}_{7} \mathrm{H}_{16}$ & 2.75 & 126.58 & 150.24 & 166.22 & 161.11 \\
\hline Pentane, 3-ethyl- & 100 & $\mathrm{C}_{7} \mathrm{H}_{16}$ & 2.85 & 90.95 & 81.43 & 107.92 & 92.06 \\
\hline 1-Heptene, 4-methyl- & 112 & $\mathrm{C}_{8} \mathrm{H}_{16}$ & 2.98 & 24.57 & 10.65 & 28.10 & 16.08 \\
\hline Cyclopentane, 1,2-dimethyl-, cis- & 98 & $\mathrm{C}_{7} \mathrm{H}_{14}$ & 3.04 & - & 16.12 & 5.37 & 6.92 \\
\hline Heptane & 100 & $\mathrm{C}_{7} \mathrm{H}_{16}$ & 3.16 & 25.22 & 23.28 & 20.41 & 17.34 \\
\hline 3-Hexene, 3-methyl-, (Z)- & 98 & $\mathrm{C}_{7} \mathrm{H}_{14}$ & 3.23 & 9.71 & 24.86 & 10.47 & 15.71 \\
\hline 2-Hexene, 3-methyl-, (Z)- & 98 & $\mathrm{C}_{7} \mathrm{H}_{14}$ & 3.34 & 2.75 & 5.31 & 2.20 & 2.32 \\
\hline 2-Pentene, 3,4-dimethyl-, (E)- & 98 & $\mathrm{C}_{7} \mathrm{H}_{14}$ & 3.4 & 2.62 & 7.96 & 2.36 & 3.79 \\
\hline Cyclohexane, methyl- & 98 & $\mathrm{C}_{7} \mathrm{H}_{14}$ & 3.53 & 11.81 & 12.59 & 14.08 & 13.02 \\
\hline Hexane, 2,5-dimethyl- & 114 & $\mathrm{C}_{8} \mathrm{H}_{18}$ & 3.62 & 16.60 & 9.73 & 13.05 & 11.17 \\
\hline Hexane, 2,4-dimethyl- & 114 & $\mathrm{C}_{8} \mathrm{H}_{18}$ & 3.66 & 24.86 & 13.81 & 21.00 & 13.60 \\
\hline Cyclopentane, 1,2,4-trimethyl- & 112 & $\mathrm{C}_{8} \mathrm{H}_{16}$ & 3.8 & 3.37 & 3.14 & 2.94 & 3.80 \\
\hline 2-Pentene, 3-ethyl-2-methyl- & 112 & $\mathrm{C}_{8} \mathrm{H}_{16}$ & 4.07 & 2.52 & 5.91 & 2.32 & 8.42 \\
\hline Hexane, 2,3-dimethyl- & 114 & $\mathrm{C}_{8} \mathrm{H}_{18}$ & 4.14 & 15.05 & 8.48 & 10.82 & 40.97 \\
\hline
\end{tabular}




\begin{tabular}{|c|c|c|c|c|c|c|c|}
\hline Compound Name & $\mathbf{M}+$ & Formula & RT & $\begin{array}{c}\text { Ext Wax } \\
\mathrm{mg} / \mathrm{g}\end{array}$ & $\begin{array}{c}\text { WO450 } \\
\text { mg/g }\end{array}$ & $\begin{array}{c}\text { WO500 } \\
\text { mg/g }\end{array}$ & $\begin{array}{c}\text { WO } 550 \\
\text { mg/g }\end{array}$ \\
\hline Heptane, 2-methyl- & 114 & $\mathrm{C}_{8} \mathrm{H}_{18}$ & 4.25 & 60.89 & 34.11 & 48.03 & 69.17 \\
\hline Toluene & 92 & $\mathrm{C}_{7} \mathrm{H}_{8}$ & 4.33 & 43.43 & 83.16 & 61.05 & 35.76 \\
\hline Heptane, 3-methyl- & 114 & $\mathrm{C}_{8} \mathrm{H}_{18}$ & 4.41 & 52.59 & 33.32 & 43.63 & 4.39 \\
\hline Cyclohexane, 1,3-dimethyl-, trans- & 112 & $\mathrm{C}_{8} \mathrm{H}_{16}$ & 4.52 & 5.61 & 2.74 & 4.00 & 3.62 \\
\hline 2-Heptene, 6-methyl- & 112 & $\mathrm{C}_{8} \mathrm{H}_{16}$ & 4.7 & - & 2.40 & - & 0.00 \\
\hline Cyclopentane, 1-ethyl-3-methyl-, cis & 112 & $\mathrm{C}_{8} \mathrm{H}_{16}$ & 4.72 & - & 1.32 & 0.74 & 1.82 \\
\hline Cyclopentane, 1-ethyl-3-methyl-, trans- & 112 & $\mathrm{C}_{8} \mathrm{H}_{16}$ & 4.79 & - & 2.40 & 1.28 & 1.72 \\
\hline Heptane, 4-methylene- & 112 & $\mathrm{C}_{8} \mathrm{H}_{16}$ & 4.83 & - & 3.77 & 1.43 & 0.90 \\
\hline 3-Octene, (Z)- & 112 & $\mathrm{C}_{8} \mathrm{H}_{16}$ & 4.9 & - & 2.38 & 0.95 & 7.70 \\
\hline Hexane, 3-ethyl- & 114 & $\mathrm{C}_{8} \mathrm{H}_{18}$ & 4.96 & 12.04 & 9.11 & 7.43 & 2.43 \\
\hline 2-Methyl-2-heptene & 112 & $\mathrm{C}_{8} \mathrm{H}_{16}$ & 5.03 & 1.60 & 3.39 & 1.60 & 3.11 \\
\hline Cyclohexane, 1,4-dimethyl- & 112 & $\mathrm{C}_{8} \mathrm{H}_{16}$ & 5.1 & 2.40 & 3.45 & 2.10 & 2.51 \\
\hline 4-Octene, $(\mathrm{E})$ - & 112 & $\mathrm{C}_{8} \mathrm{H}_{16}$ & 5.19 & 1.59 & 3.58 & 1.75 & 3.98 \\
\hline Hexane, 2,3,5-trimethyl- & 128 & $\mathrm{C}_{9} \mathrm{H}_{20}$ & 5.3 & 1.29 & 1.79 & 0.99 & 4.69 \\
\hline Heptane, 2,4-dimethyl- & 128 & $\mathrm{C}_{9} \mathrm{H}_{20}$ & 5.47 & 7.02 & 3.94 & 4.30 & 12.06 \\
\hline Octane, 2-methyl- & 128 & $\mathrm{C}_{9} \mathrm{H}_{20}$ & 5.62 & 7.39 & 3.84 & 4.57 & 0.63 \\
\hline Heptane, 3,5-dimethyl- & 128 & $\mathrm{C}_{9} \mathrm{H}_{20}$ & 5.79 & 19.62 & 9.57 & 11.70 & 1.75 \\
\hline Hexane, 3-ethyl-2-methyl- & 128 & $\mathrm{C}_{9} \mathrm{H}_{20}$ & 6.08 & 0.98 & 1.07 & 51.84 & 2.71 \\
\hline Cyclohexane, 1,2,4-trimethyl- & 126 & $\mathrm{C}_{9} \mathrm{H}_{18}$ & 6.21 & 1.02 & - & - & 0.71 \\
\hline Heptane, 2,3-dimethyl- & 128 & $\mathrm{C}_{9} \mathrm{H}_{20}$ & 6.32 & 3.71 & - & 2.20 & 27.69 \\
\hline Octane, 4-methyl- & 128 & $\mathrm{C}_{9} \mathrm{H}_{20}$ & 6.55 & 30.53 & 24.96 & 19.46 & 64.40 \\
\hline p-Xylene & 106 & $\mathrm{C}_{8} \mathrm{H}_{10}$ & 6.73 & 54.97 & 68.21 & 44.73 & 16.86 \\
\hline o-Xylene & 106 & $\mathrm{C}_{8} \mathrm{H}_{10}$ & 7.36 & 12.57 & 17.32 & 9.82 & 4.45 \\
\hline Nonane & 128 & $\mathrm{C}_{9} \mathrm{H}_{20}$ & 7.5 & 4.35 & 4.69 & 2.33 & 1.47 \\
\hline Cyclohexane, 1-ethyl-2-methyl-, cis- & 126 & $\mathrm{C}_{9} \mathrm{H}_{18}$ & 7.71 & 0.76 & 1.87 & 0.54 & 1.40 \\
\hline Octane, 4-ethyl- & 142 & $\mathrm{C}_{10} \mathrm{H}_{22}$ & 7.83 & 1.25 & - & - & - \\
\hline Heptane, 2,4,6-trimethyl- & 142 & $\mathrm{C}_{10} \mathrm{H}_{22}$ & 7.99 & 2.12 & 0.87 & 0.88 & 3.68 \\
\hline Octane, 3,5-dimethyl- & 142 & $\mathrm{C}_{10} \mathrm{H}_{22}$ & 8.2 & 5.20 & 2.62 & 2.03 & 2.76 \\
\hline Octane, 2,7-dimethyl- & 143 & $\mathrm{C}_{10} \mathrm{H}_{23}$ & 8.32 & 1.37 & 0.81 & 0.45 & 3.58 \\
\hline Octane, 3-ethyl- & 144 & $\mathrm{C}_{10} \mathrm{H}_{24}$ & 8.35 & 1.49 & 0.96 & 1.28 & 3.11 \\
\hline Octane, 2,6-dimethyl- & 142 & $\mathrm{C}_{10} \mathrm{H}_{22}$ & 8.46 & 3.69 & 2.51 & 1.62 & 3.44 \\
\hline Nonane, 3-methyl- & 143 & $\mathrm{C}_{10} \mathrm{H}_{23}$ & 8.62 & 2.96 & 2.34 & 1.27 & 1.67 \\
\hline Octane, 2,3-dimethyl- & 142 & $\mathrm{C}_{10} \mathrm{H}_{22}$ & 9.09 & 3.48 & 3.55 & 1.45 & 2.15 \\
\hline Nonane, 5-methyl- & 142 & $\mathrm{C}_{10} \mathrm{H}_{22}$ & 9.23 & 1.87 & 0.90 & 0.76 & 14.99 \\
\hline Nonane, 4-methyl- & 142 & $\mathrm{C}_{10} \mathrm{H}_{22}$ & 9.27 & 3.67 & 7.18 & 1.32 & 11.80 \\
\hline Nonane, 2-methyl- & 142 & $\mathrm{C}_{10} \mathrm{H}_{22}$ & 9.36 & 12.50 & 6.44 & 6.22 & 1.90 \\
\hline Benzene, 1,2,4-trimethyl- & 120 & $\mathrm{C}_{9} \mathrm{H}_{12}$ & 9.52 & 12.20 & 12.41 & 5.65 & 0.88 \\
\hline Benzene, 1-ethyl-3-methyl- & 121 & $\mathrm{C}_{9} \mathrm{H}_{13}$ & 9.87 & 1.41 & 2.36 & 0.71 & 18.42 \\
\hline Cyclodecane & 140 & $\mathrm{C}_{10} \mathrm{H}_{20}$ & 10 & 0.20 & 0.31 & 0.22 & 2.26 \\
\hline Mesitylene & 120 & $\mathrm{C}_{9} \mathrm{H}_{12}$ & 10.27 & 15.95 & 25.56 & 8.81 & 1.00 \\
\hline
\end{tabular}




\begin{tabular}{|c|c|c|c|c|c|c|c|}
\hline Compound Name & $\mathbf{M}+$ & Formula & RT & $\begin{array}{c}\text { Ext Wax } \\
\text { mg/g }\end{array}$ & $\begin{array}{c}\text { WO450 } \\
\mathrm{mg} / \mathrm{g}\end{array}$ & $\begin{array}{c}\text { WO500 } \\
\text { mg/g }\end{array}$ & $\begin{array}{c}\text { WO } 550 \\
\mathrm{mg} / \mathrm{g}\end{array}$ \\
\hline Decane & 140 & $\mathrm{C}_{10} \mathrm{H}_{20}$ & 10.41 & 1.88 & 2.56 & 0.68 & 1.19 \\
\hline 5-Decene & 140 & $\mathrm{C}_{10} \mathrm{H}_{20}$ & 10.59 & - & 1.19 & - & 1.03 \\
\hline Octane, 5-ethyl-2-methyl- & 156 & $\mathrm{C}_{11} \mathrm{H}_{24}$ & 10.82 & 1.25 & 1.18 & 0.43 & 2.68 \\
\hline Decane, 4-methyl- & 156 & $\mathrm{C}_{11} \mathrm{H}_{24}$ & 11.01 & 1.20 & 1.07 & 0.40 & 1.92 \\
\hline Nonane, 2,5-dimethyl- & 157 & $\mathrm{C}_{11} \mathrm{H}_{25}$ & 11.09 & 1.02 & - & 1.51 & 1.58 \\
\hline Benzene, 1,2,3-trimethyl- & 120 & $\mathrm{C}_{9} \mathrm{H}_{12}$ & 11.14 & 2.53 & 5.04 & 2.83 & 1.26 \\
\hline Nonane, 3,7-dimethyl- & 156 & $\mathrm{C}_{11} \mathrm{H}_{24}$ & 11.24 & 2.03 & 1.71 & 0.65 & 0.98 \\
\hline Decane, 2,9-dimethyl- & 170 & $\mathrm{C}_{12} \mathrm{H}_{26}$ & 11.44 & 1.59 & 1.59 & 0.55 & 1.42 \\
\hline Decane, 3-methyl- & 156 & $\mathrm{C}_{11} \mathrm{H}_{24}$ & 11.57 & 1.08 & 2.27 & 0.44 & 2.50 \\
\hline Benzene, 1-methyl-3-propyl- & 134 & $\mathrm{C}_{10} \mathrm{H}_{14}$ & 12 & 1.40 & 2.43 & 1.64 & 3.92 \\
\hline Decane, 5-methyl- & 156 & $\mathrm{C}_{11} \mathrm{H}_{24}$ & 12.13 & 2.07 & 2.89 & 0.74 & 2.06 \\
\hline Benzene, 2-ethyl-1,4-dimethyl- & 134 & $\mathrm{C}_{10} \mathrm{H}_{14}$ & 12.22 & 3.73 & 5.22 & 1.42 & 0.23 \\
\hline Decane, 2-methyl- & 156 & $\mathrm{C}_{11} \mathrm{H}_{24}$ & 12.33 & 2.09 & 2.48 & - & 2.09 \\
\hline Undecane, 4-methyl- & 170 & $\mathrm{C}_{12} \mathrm{H}_{26}$ & 12.41 & 0.25 & 0.46 & 0.70 & 1.24 \\
\hline o-Cymene & 134 & $\mathrm{C}_{10} \mathrm{H}_{14}$ & 12.78 & 1.34 & 2.58 & 0.52 & 1.90 \\
\hline Benzene, 4-ethyl-1,2-dimethyl- & 134 & $\mathrm{C}_{10} \mathrm{H}_{14}$ & 12.83 & 0.93 & 1.59 & 0.40 & 0.42 \\
\hline Benzene, 2-ethyl-1,3-dimethyl- & 134 & $\mathrm{C}_{10} \mathrm{H}_{14}$ & 13.02 & 1.69 & 3.79 & 0.67 & 0.83 \\
\hline Undecane & 156 & $\mathrm{C}_{11} \mathrm{H}_{24}$ & 13.4 & 0.97 & 1.18 & 0.30 & 0.48 \\
\hline Cyclopropane, 1-pentyl-2-propyl- & 154 & $\mathrm{C}_{11} \mathrm{H}_{22}$ & 13.57 & - & 1.31 & - & 1.78 \\
\hline Benzene, 1,2,4,5-tetramethyl- & 134 & $\mathrm{C}_{10} \mathrm{H}_{14}$ & 13.93 & 2.69 & 3.47 & 1.07 & 2.03 \\
\hline Benzene, 1-ethyl-3,5-dimethyl- & 134 & $\mathrm{C}_{10} \mathrm{H}_{14}$ & 14.06 & 2.86 & 4.15 & 1.05 & 0.53 \\
\hline Benzene, 2-ethenyl-1,4-dimethyl- & 132 & $\mathrm{C}_{10} \mathrm{H}_{12}$ & 14.96 & - & 1.15 & 0.24 & 0.36 \\
\hline Benzene, 1-ethyl-2,3-dimethyl- & 134 & $\mathrm{C}_{10} \mathrm{H}_{14}$ & 15.01 & 1.76 & 2.63 & 0.75 & 1.70 \\
\hline 2,3-Dimethyldecane & 170 & $\mathrm{C}_{12} \mathrm{H}_{26}$ & 15.15 & 0.81 & 1.38 & 0.27 & 0.51 \\
\hline Undecane, 2-methyl- & 170 & $\mathrm{C}_{12} \mathrm{H}_{26}$ & 15.27 & 1.11 & 1.33 & 0.40 & 0.56 \\
\hline Undecane, 3-methyl- & 170 & $\mathrm{C}_{12} \mathrm{H}_{26}$ & 15.47 & 0.84 & 1.18 & 0.33 & 0.49 \\
\hline Benzene, 1-methyl-4-(1-methylpropyl)- & 148 & $\mathrm{C}_{11} \mathrm{H}_{16}$ & 15.66 & - & 0.54 & 0.66 & 1.85 \\
\hline 1H-Indene, 1-methylene- & 128 & $\mathrm{C}_{10} \mathrm{H}_{8}$ & 15.98 & - & 1.17 & - & - \\
\hline Benzene, pentamethyl- & 148 & $\mathrm{C}_{11} \mathrm{H}_{16}$ & 16.32 & 1.20 & 1.95 & 0.44 & 1.61 \\
\hline 1H-Indene, 1-ethylidene- & 142 & $\mathrm{C}_{11} \mathrm{H}_{10}$ & 19.08 & - & 1.13 & - & - \\
\hline
\end{tabular}


Table S3. Identified compounds in gasoline and the liquid products of thermal pyrolysis of solvent extracted wax and wax-oil samples collected from the U-tube. The units are mg compound per g resultant liquid product.

\begin{tabular}{|c|c|c|c|c|c|c|c|}
\hline Compound Name & M+ & Formula & $\begin{array}{l}\text { RT } \\
\text { min }\end{array}$ & $\begin{array}{c}\text { ExtWax } \\
\mathrm{mg} / \mathrm{g}\end{array}$ & $\begin{array}{c}\text { WO450 } \\
\mathrm{mg} / \mathrm{g}\end{array}$ & $\begin{array}{c}\text { WO500 } \\
\mathrm{mg} / \mathrm{g}\end{array}$ & $\begin{array}{c}\text { WO550 } \\
\mathrm{mg} / \mathrm{g}\end{array}$ \\
\hline 1-Methylpentyl cyclopropane & 126 & $\mathrm{C}_{9} \mathrm{H}_{18}$ & 3.06 & 0.12 & - & - & - \\
\hline Propanoic acid & 74 & $\mathrm{C}_{3} \mathrm{H}_{6} \mathrm{O}_{2}$ & 3.14 & - & 0.47 & 0.57 & 2.32 \\
\hline Acetic acid, hydroxy-, methyl ester & 90 & $\mathrm{C}_{3} \mathrm{H}_{6} \mathrm{O}_{3}$ & 3.25 & - & 0.07 & 0.34 & - \\
\hline Acetoin & 88 & $\mathrm{C}_{4} \mathrm{H}_{8} \mathrm{O}_{2}$ & 3.41 & - & 0.17 & 0.19 & 0.46 \\
\hline 1-Propanol & 60 & $\mathrm{C}_{3} \mathrm{H}_{8} \mathrm{O}$ & 3.72 & - & 0.44 & 0.42 & 0.51 \\
\hline Propanoic acid, 2-methyl- & 88 & $\mathrm{C}_{4} \mathrm{H}_{8} \mathrm{O}_{2}$ & 4.1 & - & - & - & 0.23 \\
\hline 2,2-Dimethoxybutane & 118 & $\mathrm{C}_{6} \mathrm{H}_{14} \mathrm{O}_{2}$ & 4.2 & 0.20 & 0.26 & 0.64 & 0.53 \\
\hline Cyclobutene, 2-propenylidene- & 92 & $\mathrm{C}_{7} \mathrm{H}_{8}$ & 4.35 & - & 0.70 & 0.41 & 1.03 \\
\hline Toluene & 92 & $\mathrm{C}_{7} \mathrm{H}_{8}$ & 4.41 & - & 0.89 & 1.10 & 0.36 \\
\hline Cyclopentanol & 86 & $\mathrm{C}_{5} \mathrm{H}_{10} \mathrm{O}$ & 4.69 & - & 1.96 & 2.76 & 3.04 \\
\hline Cyclopropane, pentyl- & 112 & $\mathrm{C}_{8} \mathrm{H}_{16}$ & 4.8 & 1.45 & - & - & - \\
\hline 1-Methyl-2-methylenecyclohexane & 110 & $\mathrm{C}_{8} \mathrm{H}_{14}$ & 4.86 & - & 0.33 & - & 1.00 \\
\hline 3,3-Dimethylcyclohexanol & 128 & $\mathrm{C}_{8} \mathrm{H}_{16} \mathrm{O}$ & 5.06 & - & 0.34 & - & - \\
\hline Cyclopropane, pentyl- & 112 & $\mathrm{C}_{8} \mathrm{H}_{16}$ & 5.58 & - & 0.50 & 0.18 & 0.65 \\
\hline 1,4-Cyclohexanediol, trans- & 116 & $\mathrm{C}_{6} \mathrm{H}_{12} \mathrm{O}_{2}$ & 5.89 & - & 0.48 & 0.43 & 2.26 \\
\hline 1,2-Ethanediol, monoacetate & 104 & $\mathrm{C}_{4} \mathrm{H}_{8} \mathrm{O}_{3}$ & 6.29 & - & 0.24 & - & - \\
\hline 3-Furanmethanol & 98 & $\mathrm{C}_{5} \mathrm{H}_{6} \mathrm{O}_{2}$ & 6.42 & - & 0.33 & 0.19 & 0.35 \\
\hline 2-Butanone & 72 & $\mathrm{C}_{4} \mathrm{H}_{8} \mathrm{O}$ & 6.49 & - & 0.52 & 0.55 & 0.78 \\
\hline 2-Propanone, 1-(acetyloxy)- & 116 & $\mathrm{C}_{5} \mathrm{H}_{8} \mathrm{O}_{3}$ & 6.77 & - & 1.11 & 0.07 & 1.63 \\
\hline p-Xylene & 106 & $\mathrm{C}_{8} \mathrm{H}_{10}$ & 6.81 & - & 0.28 & 0.14 & - \\
\hline 1-Nonene & 126 & $\mathrm{C}_{9} \mathrm{H}_{18}$ & 7.29 & 3.77 & 0.15 & - & 0.41 \\
\hline Nonane & 128 & $\mathrm{C}_{9} \mathrm{H}_{20}$ & 7.52 & 0.25 & 0.28 & 0.25 & - \\
\hline 1,2,3-Cyclopentanetriol & 118 & $\mathrm{C}_{5} \mathrm{H}_{10} \mathrm{O}_{3}$ & 7.69 & - & - & - & 0.24 \\
\hline 2-Cyclopenten-1-one, 2-methyl- & 96 & $\mathrm{C}_{6} \mathrm{H}_{8} \mathrm{O}$ & 7.79 & - & - & - & 1.61 \\
\hline 2-Methyl-2-cyclopentenone & 96 & $\mathrm{C}_{6} \mathrm{H}_{8} \mathrm{O}$ & 7.82 & - & 0.29 & 0.33 & - \\
\hline Ethanone, 1-(2-furanyl)- & 110 & $\mathrm{C}_{6} \mathrm{H}_{6} \mathrm{O}_{2}$ & 7.93 & - & 0.25 & 0.17 & 0.52 \\
\hline Acetylfuran & 110 & $\mathrm{C}_{6} \mathrm{H}_{6} \mathrm{O}_{2}$ & 8.05 & - & 3.32 & 1.22 & 1.48 \\
\hline 2-Cyclopenten-1-one, 2-hydroxy- & 98 & $\mathrm{C}_{5} \mathrm{H}_{6} \mathrm{O}_{2}$ & 8.28 & - & 0.46 & 0.15 & - \\
\hline 2,5-Hexanedione & 114 & $\mathrm{C}_{6} \mathrm{H}_{10} \mathrm{O}_{2}$ & 8.42 & - & 0.43 & 0.12 & 0.42 \\
\hline 2-Cyclohexen-1-one & 96 & $\mathrm{C}_{6} \mathrm{H}_{8} \mathrm{O}$ & 8.52 & 9.50 & 0.16 & - & 0.30 \\
\hline 2(5H)-Furanone, 5-methyl- & 98 & $\mathrm{C}_{5} \mathrm{H}_{6} \mathrm{O}_{2}$ & 8.77 & 0.50 & 0.24 & 0.14 & 0.19 \\
\hline 2(3H)-Furanone, dihydro-5-methyl- & 100 & $\mathrm{C}_{5} \mathrm{H}_{8} \mathrm{O}_{2}$ & 9.18 & - & 0.28 & - & 0.12 \\
\hline 2-Cyclopenten-1-one, 3-methyl- & 96 & $\mathrm{C}_{6} \mathrm{H}_{8} \mathrm{O}$ & 9.47 & - & 1.40 & - & 3.74 \\
\hline
\end{tabular}




\begin{tabular}{|c|c|c|c|c|c|c|c|}
\hline Compound Name & $\mathbf{M}+$ & Formula & $\begin{array}{l}\text { RT } \\
\min \end{array}$ & $\begin{array}{c}\text { ExtWax } \\
\mathrm{mg} / \mathrm{g}\end{array}$ & $\begin{array}{c}\text { WO450 } \\
\mathrm{mg} / \mathrm{g}\end{array}$ & $\begin{array}{c}\text { WO500 } \\
\mathrm{mg} / \mathrm{g}\end{array}$ & $\begin{array}{c}\text { WO550 } \\
\mathrm{mg} / \mathrm{g}\end{array}$ \\
\hline 5-Methyl furfural & 110 & $\mathrm{C}_{6} \mathrm{H}_{6} \mathrm{O}_{2}$ & 9.53 & - & - & 1.17 & - \\
\hline Phenol & 94 & $\mathrm{C}_{6} \mathrm{H}_{6} \mathrm{O}$ & 9.97 & - & 2.48 & 1.30 & 4.06 \\
\hline 1-Decene & 140 & $\mathrm{C}_{10} \mathrm{H}_{20}$ & 10.18 & - & 1.59 & 0.92 & 3.24 \\
\hline Cyclohexanol, 2,3-dimethyl- & 128 & $\mathrm{C}_{8} \mathrm{H}_{16} \mathrm{O}$ & 10.26 & - & 0.49 & 0.22 & 0.52 \\
\hline Decane & 142 & $\mathrm{C}_{10} \mathrm{H}_{22}$ & 10.44 & - & - & - & - \\
\hline Octanal & 128 & $\mathrm{C}_{8} \mathrm{H}_{16} \mathrm{O}$ & 10.58 & 17.87 & 0.48 & 0.35 & 0.44 \\
\hline 2-Cyclohexen-1-one, 4,4-dimethyl- & 124 & $\mathrm{C}_{8} \mathrm{H}_{12} \mathrm{O}$ & 11 & - & - & - & 0.30 \\
\hline 2,4-Hexadiene, 2,5-dimethyl- & 110 & $\mathrm{C}_{8} \mathrm{H}_{14}$ & 11.16 & - & - & - & 0.39 \\
\hline 1,2-Cyclopentanedione, 3-methyl- & 112 & $\mathrm{C}_{6} \mathrm{H}_{8} \mathrm{O}_{2}$ & 11.32 & 1.07 & 3.85 & 3.84 & 2.06 \\
\hline 2-Cyclopenten-1-one, 2,3-dimethyl- & 110 & $\mathrm{C}_{7} \mathrm{H}_{10} \mathrm{O}$ & 11.68 & - & 0.82 & 0.56 & 1.58 \\
\hline 1,4-Cyclohex-2-enedione & 110 & $\mathrm{C}_{6} \mathrm{H}_{6} \mathrm{O}_{2}$ & 11.87 & - & 1.44 & 1.92 & 0.51 \\
\hline 3-Methyl-3-buten-1-ol & 86 & $\mathrm{C}_{5} \mathrm{H}_{10} \mathrm{O}$ & 11.95 & - & - & - & 0.34 \\
\hline Phenol, 2-methyl- & 108 & $\mathrm{C}_{7} \mathrm{H}_{8} \mathrm{O}$ & 12.15 & - & 2.16 & 1.55 & 4.30 \\
\hline 2-Furanol, tetrahydro-2-methyl- & 102 & $\mathrm{C}_{5} \mathrm{H}_{10} \mathrm{O}_{2}$ & 12.47 & 13.17 & 1.06 & 0.09 & 0.41 \\
\hline Acetophenone & 120 & $\mathrm{C}_{8} \mathrm{H}_{8} \mathrm{O}$ & 12.53 & - & - & - & 0.51 \\
\hline Phenol, 3-methyl- & 108 & $\mathrm{C}_{7} \mathrm{H}_{8} \mathrm{O}$ & 12.77 & 0.44 & 1.53 & 0.13 & 3.54 \\
\hline 1,10-Undecadiene & 152 & $\mathrm{C}_{11} \mathrm{H}_{20}$ & 12.94 & 20.58 & 0.42 & 1.11 & 0.34 \\
\hline 1-Undecene & 154 & $\mathrm{C}_{11} \mathrm{H}_{22}$ & 13.18 & - & 9.44 & 0.39 & 0.29 \\
\hline Undecane & 156 & $\mathrm{C}_{11} \mathrm{H}_{24}$ & 13.42 & 1.12 & 3.44 & 4.98 & 14.43 \\
\hline 2-Propylcyclohexanol & 142 & $\mathrm{C}_{9} \mathrm{H}_{18} \mathrm{O}$ & 13.59 & - & - & - & 0.85 \\
\hline Nonanal & 142 & $\mathrm{C}_{9} \mathrm{H}_{18} \mathrm{O}$ & 13.61 & - & 0.85 & 1.13 & 0.91 \\
\hline Phenol, 2,3-dimethyl- & 122 & $\mathrm{C}_{8} \mathrm{H}_{10} \mathrm{O}$ & 13.7 & - & - & - & 0.81 \\
\hline Maltol & 126 & $\mathrm{C}_{6} \mathrm{H}_{6} \mathrm{O}_{3}$ & 13.89 & - & 0.72 & 0.41 & 0.46 \\
\hline 2-Cyclopenten-1-one, 3-ethyl-2-hydroxy- & 126 & $\mathrm{C}_{7} \mathrm{H}_{10} \mathrm{O}_{2}$ & 14.05 & - & 0.61 & 0.56 & 0.37 \\
\hline Phenol, 2,4-dimethyl- & 122 & $\mathrm{C}_{8} \mathrm{H}_{10} \mathrm{O}$ & 14.62 & - & - & - & 0.46 \\
\hline Phenol, 3,5-dimethyl- & 122 & $\mathrm{C}_{8} \mathrm{H}_{10} \mathrm{O}$ & 15.06 & 0.53 & 1.12 & 0.12 & 1.24 \\
\hline Phenol, 2-ethyl- & 122 & $\mathrm{C}_{8} \mathrm{H}_{10} \mathrm{O}$ & 15.53 & - & 0.45 & 0.82 & 2.43 \\
\hline 1,11-Dodecadiene & 166 & $\mathrm{C}_{12} \mathrm{H}_{22}$ & 15.86 & 0.97 & - & - & 1.18 \\
\hline 1-Dodecene & 168 & $\mathrm{C}_{12} \mathrm{H}_{24}$ & 16.1 & - & 13.12 & 7.06 & 21.88 \\
\hline 3-Dodecene, (Z)- & 168 & $\mathrm{C}_{12} \mathrm{H}_{24}$ & 16.25 & 0.64 & 1.40 & 1.50 & 0.79 \\
\hline Dodecane & 170 & $\mathrm{C}_{12} \mathrm{H}_{26}$ & 16.34 & 15.54 & 0.97 & 0.53 & 0.72 \\
\hline Catechol & 110 & $\mathrm{C}_{6} \mathrm{H}_{6} \mathrm{O}_{2}$ & 16.41 & - & - & - & 0.26 \\
\hline Decanal & 156 & $\mathrm{C}_{10} \mathrm{H}_{20} \mathrm{O}$ & 16.55 & 0.77 & 1.03 & - & - \\
\hline 1,4:3,6-Dianhydro- $\alpha$-d-glucopyranose & 144 & $\mathrm{C}_{6} \mathrm{H}_{8} \mathrm{O}_{4}$ & 16.75 & - & 0.68 & 0.91 & 0.21 \\
\hline 3,4-Anhydro-d-galactosan & 144 & $\mathrm{C}_{6} \mathrm{H}_{8} \mathrm{O}_{4}$ & 17.08 & - & - & 0.47 & - \\
\hline 2,3-Anhydro-d-mannosan & 144 & $\mathrm{C}_{6} \mathrm{H}_{8} \mathrm{O}_{4}$ & 17.24 & - & - & 0.99 & - \\
\hline Benzene, 1-ethyl-4-methoxy- & 136 & $\mathrm{C}_{9} \mathrm{H}_{12} \mathrm{O}$ & 17.49 & - & - & - & 0.36 \\
\hline Dihydrocarveol & 154 & $\mathrm{C}_{10} \mathrm{H}_{18} \mathrm{O}$ & 18.09 & - & - & - & 0.75 \\
\hline
\end{tabular}




\begin{tabular}{|c|c|c|c|c|c|c|c|}
\hline Compound Name & $\mathbf{M}+$ & Formula & $\begin{array}{l}\text { RT } \\
\min \end{array}$ & $\begin{array}{c}\text { ExtWax } \\
\mathrm{mg} / \mathrm{g}\end{array}$ & $\begin{array}{c}\text { WO450 } \\
\mathrm{mg} / \mathrm{g}\end{array}$ & $\begin{array}{c}\text { WO500 } \\
\mathrm{mg} / \mathrm{g}\end{array}$ & $\begin{array}{c}\text { WO550 } \\
\mathrm{mg} / \mathrm{g}\end{array}$ \\
\hline (3E)-Trideca-1,3-diene & 180 & $\mathrm{C}_{13} \mathrm{H}_{24}$ & 18.68 & - & 0.83 & 0.94 & 1.15 \\
\hline 1-Tridecene & 182 & $\mathrm{C}_{13} \mathrm{H}_{26}$ & 18.89 & 0.25 & 13.90 & 12.04 & 21.55 \\
\hline 2-Undecanone, 6,10-dimethyl- & 198 & $\mathrm{C}_{13} \mathrm{H}_{26} \mathrm{O}$ & 18.97 & - & 0.53 & - & - \\
\hline Tridecane & 184 & $\mathrm{C}_{13} \mathrm{H}_{28}$ & 19.11 & 12.19 & 1.13 & 0.82 & 1.11 \\
\hline Undecanal & 170 & $\mathrm{C}_{11} \mathrm{H}_{22} \mathrm{O}$ & 19.37 & 0.78 & 0.89 & 0.16 & - \\
\hline Phenol, 2,6-dimethoxy- & 154 & $\mathrm{C}_{8} \mathrm{H}_{10} \mathrm{O}_{3}$ & 20.63 & - & 0.50 & 0.12 & - \\
\hline 2,3-Dimethoxyphenol & 154 & $\mathrm{C}_{8} \mathrm{H}_{10} \mathrm{O}_{3}$ & 20.66 & - & - & 0.27 & - \\
\hline Phenol, 2-methoxy-4-(1-propenyl)- & 164 & $\mathrm{C}_{10} \mathrm{H}_{12} \mathrm{O}_{2}$ & 20.77 & - & 0.41 & 0.37 & - \\
\hline 1,13-Tetradecadiene & 194 & $\mathrm{C}_{14} \mathrm{H}_{26}$ & 21.35 & 0.27 & 0.63 & 0.41 & 1.31 \\
\hline 1-Tetradecene & 196 & $\mathrm{C}_{14} \mathrm{H}_{28}$ & 21.55 & 9.79 & 13.41 & 15.10 & 22.00 \\
\hline 2-Dodecanone & 184 & $\mathrm{C}_{12} \mathrm{H}_{24} \mathrm{O}$ & 21.67 & 0.51 & 0.44 & - & - \\
\hline Tetradecane & 198 & $\mathrm{C}_{14} \mathrm{H}_{30}$ & 21.75 & - & 0.85 & 0.67 & 0.75 \\
\hline Dodecanal & 184 & $\mathrm{C}_{12} \mathrm{H}_{24} \mathrm{O}$ & 22.03 & - & 0.70 & 0.68 & - \\
\hline Isoeugenol & 164 & $\mathrm{C}_{10} \mathrm{H}_{12} \mathrm{O}_{2}$ & 23.16 & - & 0.91 & 4.74 & 0.70 \\
\hline Pentadecadiene & 208 & $\mathrm{C}_{15} \mathrm{H}_{28}$ & 23.88 & 0.22 & 1.20 & 13.60 & 1.55 \\
\hline 1-Pentadecene & 210 & $\mathrm{C}_{15} \mathrm{H}_{30}$ & 24.06 & 8.42 & 10.79 & 0.86 & 22.12 \\
\hline Pentadecane & 212 & $\mathrm{C}_{15} \mathrm{H}_{32}$ & 24.24 & 0.54 & 1.05 & 0.49 & 0.70 \\
\hline Tridecanal & 198 & $\mathrm{C}_{13} \mathrm{H}_{26} \mathrm{O}$ & 24.55 & - & 0.63 & - & - \\
\hline (Z)6-Pentadecen-1-ol & 226 & $\mathrm{C}_{15} \mathrm{H}_{30} \mathrm{O}$ & 25.33 & 0.16 & 0.33 & - & 0.44 \\
\hline Hexadecadiene & 222 & $\mathrm{C}_{16} \mathrm{H}_{30}$ & 26.27 & 8.14 & 0.60 & - & 1.11 \\
\hline 1-Hexadecene & 224 & $\mathrm{C}_{16} \mathrm{H}_{32}$ & 26.44 & 0.53 & 8.77 & 10.20 & 21.05 \\
\hline Hexadecane & 226 & $\mathrm{C}_{16} \mathrm{H}_{34}$ & 26.6 & 0.13 & 1.17 & 0.57 & 0.57 \\
\hline Pentadecanal & 226 & $\mathrm{C}_{15} \mathrm{H}_{30} \mathrm{O}$ & 26.94 & 7.81 & 0.40 & - & - \\
\hline 13-Heptadecyn-1-ol & 252 & $\mathrm{C}_{17} \mathrm{H}_{32} \mathrm{O}$ & 27.73 & 0.84 & 0.63 & - & - \\
\hline 1-Hexadecanol & 242 & $\mathrm{C}_{16} \mathrm{H}_{34} \mathrm{O}$ & 27.88 & 7.57 & - & - & - \\
\hline n-Heptadecadiene & 236 & $\mathrm{C}_{17} \mathrm{H}_{32}$ & 28.54 & 1.80 & 0.65 & - & 0.97 \\
\hline 1-Heptadecene & 238 & $\mathrm{C}_{17} \mathrm{H}_{34}$ & 28.69 & 7.16 & 7.67 & 8.38 & 21.02 \\
\hline Heptadecane & 240 & $\mathrm{C}_{17} \mathrm{H}_{36}$ & 28.84 & 5.49 & 0.88 & 0.64 & 0.69 \\
\hline Oxirane, tetradecyl- & 240 & $\mathrm{C}_{16} \mathrm{H}_{32} \mathrm{O}$ & 29.21 & 6.61 & 0.34 & - & - \\
\hline Octadecadiene-1,17 & 250 & $\mathrm{C}_{18} \mathrm{H}_{34}$ & 30.7 & 16.26 & 0.23 & - & 0.65 \\
\hline 1-Octadecene & 252 & $\mathrm{C}_{18} \mathrm{H}_{36}$ & 30.83 & 5.78 & 7.39 & 7.24 & 21.60 \\
\hline Octadecane & 254 & $\mathrm{C}_{18} \mathrm{H}_{38}$ & 30.98 & 45.84 & 0.69 & 0.57 & 0.65 \\
\hline 8,11-Nonadecadiene & 264 & $\mathrm{C}_{19} \mathrm{H}_{36}$ & 32.75 & 4.94 & 0.19 & - & 0.82 \\
\hline 1-Nonadecene & 266 & $\mathrm{C}_{19} \mathrm{H}_{38}$ & 32.87 & 82.66 & 7.02 & 6.55 & 19.87 \\
\hline Nonadecane & 268 & $\mathrm{C}_{19} \mathrm{H}_{40}$ & 33 & 109.92 & 0.90 & 0.87 & 1.39 \\
\hline 1-Eicosene & 280 & $\mathrm{C}_{20} \mathrm{H}_{40}$ & 34.83 & 3.24 & 6.66 & 5.81 & 19.14 \\
\hline Eicosane & 282 & $\mathrm{C}_{20} \mathrm{H}_{42}$ & 34.94 & 106.88 & 1.04 & 2.41 & 2.36 \\
\hline 1-Heneicosene & 294 & $\mathrm{C}_{21} \mathrm{H}_{42}$ & 36.69 & 2.38 & 6.18 & 5.22 & 16.49 \\
\hline
\end{tabular}




\begin{tabular}{|c|c|c|c|c|c|c|c|}
\hline Compound Name & $\mathbf{M}+$ & Formula & $\begin{array}{l}\text { RT } \\
\min \end{array}$ & $\begin{array}{c}\text { ExtWax } \\
\mathrm{mg} / \mathrm{g}\end{array}$ & $\begin{array}{c}\text { WO450 } \\
\mathrm{mg} / \mathrm{g}\end{array}$ & $\begin{array}{c}\text { WO500 } \\
\mathrm{mg} / \mathrm{g}\end{array}$ & $\begin{array}{c}\text { WO550 } \\
\mathrm{mg} / \mathrm{g}\end{array}$ \\
\hline Heneicosane & 296 & $\mathrm{C}_{21} \mathrm{H}_{44}$ & 36.79 & 1.24 & 1.95 & 6.96 & 6.14 \\
\hline 1-Docosene & 308 & $\mathrm{C}_{22} \mathrm{H}_{44}$ & 38.47 & 116.06 & 5.96 & 4.61 & 13.77 \\
\hline Docosane & 310 & $\mathrm{C}_{22} \mathrm{H}_{46}$ & 38.57 & 3.52 & 4.23 & 18.70 & 16.85 \\
\hline 1-Tricosene & 322 & $\mathrm{C}_{23} \mathrm{H}_{46}$ & 40.17 & 1.41 & 5.51 & 3.93 & 11.33 \\
\hline Tricosane & 324 & $\mathrm{C}_{23} \mathrm{H}_{48}$ & 40.28 & 96.59 & 10.21 & 48.74 & 41.47 \\
\hline 1-Tetracosene & 336 & $\mathrm{C}_{24} \mathrm{H}_{48}$ & 41.82 & 3.34 & 5.33 & 3.03 & 8.21 \\
\hline Tetracosane & 338 & $\mathrm{C}_{24} \mathrm{H}_{50}$ & 41.91 & 2.28 & 22.20 & 84.48 & 70.34 \\
\hline 6,9-Pentacosadiene & 348 & $\mathrm{C}_{25} \mathrm{H}_{48}$ & 42.89 & - & - & - & 1.36 \\
\hline 9-Pentacosene & 350 & $\mathrm{C}_{25} \mathrm{H}_{50}$ & 43.4 & - & - & - & 6.15 \\
\hline 2-Methyltetracosane & 352 & $\mathrm{C}_{25} \mathrm{H}_{52}$ & 43.49 & 81.62 & 41.77 & 118.95 & 91.96 \\
\hline 9,10-Hexacosadiene & 362 & $\mathrm{C}_{26} \mathrm{H}_{50}$ & 44.42 & 3.38 & 1.02 & 3.85 & 2.66 \\
\hline 1-Hexacosene & 364 & $\mathrm{C}_{26} \mathrm{H}_{52}$ & 45 & 1.24 & 51.99 & 108.41 & 91.54 \\
\hline 2-Methylhexacosane & 380 & $\mathrm{C}_{27} \mathrm{H}_{56}$ & 45.89 & - & 1.26 & 3.39 & 1.95 \\
\hline Heptacosadiene & 376 & $\mathrm{C}_{27} \mathrm{H}_{52}$ & 46.05 & 61.97 & 1.96 & 3.39 & 1.44 \\
\hline 1-Heptacosene & 378 & $\mathrm{C}_{27} \mathrm{H}_{54}$ & 46.25 & 2.43 & 1.61 & 1.69 & 0.93 \\
\hline Heptacosane & 380 & $\mathrm{C}_{27} \mathrm{H}_{56}$ & 46.46 & 1.31 & 70.91 & 110.21 & 86.41 \\
\hline Octacosadiene & 390 & $\mathrm{C}_{28} \mathrm{H}_{58}$ & 47.33 & 42.06 & 3.85 & 4.18 & 2.39 \\
\hline 1-Octacosene & 392 & $\mathrm{C}_{28} \mathrm{H}_{58}$ & 47.7 & 2.98 & - & 1.78 & 1.13 \\
\hline Octacosane & 394 & $\mathrm{C}_{28} \mathrm{H}_{58}$ & 47.86 & 2.36 & 74.87 & 93.85 & 67.46 \\
\hline Nonacosadiene & 404 & $\mathrm{C}_{29} \mathrm{H}_{56}$ & 48.69 & 28.20 & 4.28 & 3.77 & 1.88 \\
\hline 1-Nonacosene & 406 & $\mathrm{C}_{29} \mathrm{H}_{58}$ & 48.85 & 17.63 & 3.47 & 2.97 & 1.07 \\
\hline Nonacosane & 408 & $\mathrm{C}_{29} \mathrm{H}_{60}$ & 49.21 & 0.75 & 69.98 & 85.44 & 57.61 \\
\hline Triacontadiene & 418 & $\mathrm{C}_{30} \mathrm{H}_{60}$ & 50.02 & 0.59 & 4.68 & 3.63 & 1.78 \\
\hline 1-Triacontene & 420 & $\mathrm{C}_{30} \mathrm{H}_{60}$ & 50.17 & 12.17 & 2.37 & 2.43 & - \\
\hline Triacontane & 422 & $\mathrm{C}_{30} \mathrm{H}_{62}$ & 50.51 & 7.42 & 52.80 & 64.21 & 44.58 \\
\hline Hentriaconta-1,3-diene & 432 & $\mathrm{C}_{31} \mathrm{H}_{60}$ & 51.31 & 4.81 & 3.93 & 3.28 & 1.32 \\
\hline 1-Hentriacontene & 434 & $\mathrm{C}_{31} \mathrm{H}_{62}$ & 51.46 & 2.10 & - & 2.83 & 0.95 \\
\hline Hentriacontane & 436 & $\mathrm{C}_{31} \mathrm{H}_{64}$ & 51.78 & 1.22 & 41.02 & 46.30 & 32.66 \\
\hline Dotriacontadiene & 446 & $\mathrm{C}_{32} \mathrm{H}_{62}$ & 51.92 & - & 5.89 & - & - \\
\hline 1-Dotriacontene & 448 & $\mathrm{C}_{32} \mathrm{H}_{64}$ & 52.56 & - & 4.70 & 3.99 & - \\
\hline Dotriacontane & 450 & $\mathrm{C}_{32} \mathrm{H}_{66}$ & 53.02 & - & 30.73 & 34.25 & - \\
\hline 1-Tritriacontene & 462 & $\mathrm{C}_{33} \mathrm{H}_{66}$ & 54.21 & - & 2.66 & 24.01 & - \\
\hline Tritriacontane & 464 & $\mathrm{C}_{33} \mathrm{H}_{68}$ & 54.42 & - & 21.43 & - & 0.95 \\
\hline 1-Tetratriacontene & 476 & $\mathrm{C}_{34} \mathrm{H}_{68}$ & 55.08 & - & 2.29 & 0.82 & 0.71 \\
\hline Tetratriacontane & 478 & $\mathrm{C}_{34} \mathrm{H}_{70}$ & 55.37 & - & 14.40 & 16.80 & 7.28 \\
\hline Pentatriacontane & 492 & $\mathrm{C}_{35} \mathrm{H}_{72}$ & 56.5 & - & 10.48 & 11.40 & - \\
\hline Hexatriacontane & 507 & $\mathrm{C}_{36} \mathrm{H}_{74}$ & 57.6 & - & 6.50 & 8.78 & 5.33 \\
\hline Heptatriacontane & 521 & $\mathrm{C}_{37} \mathrm{H}_{76}$ & 58.84 & - & 4.53 & 5.65 & 2.38 \\
\hline
\end{tabular}




\begin{tabular}{|l|c|c|c|c|c|c|c|}
\hline Compound Name & $\mathbf{M}+$ & Formula & $\begin{array}{c}\text { RT } \\
\mathbf{m i n}\end{array}$ & $\begin{array}{c}\text { ExtWax } \\
\mathbf{m g} / \mathbf{g}\end{array}$ & $\begin{array}{c}\text { WO450 } \\
\mathbf{m g} / \mathbf{g}\end{array}$ & $\begin{array}{c}\mathbf{W O 5 0 0} \\
\mathbf{m g} / \mathbf{g}\end{array}$ & $\begin{array}{c}\text { WO550 } \\
\mathbf{m g} / \mathbf{g}\end{array}$ \\
\hline Octatriacontane & 535 & $\mathrm{C}_{38} \mathrm{H}_{78}$ & 60.23 & - & 2.55 & 3.01 & 1.46 \\
\hline 13,25-Dimethylheptatriacontane & 549 & $\mathrm{C}_{39} \mathrm{H}_{80}$ & 61.84 & - & 1.33 & 1.39 & - \\
\hline
\end{tabular}

Table S4. Identified compounds in gasoline and the liquid products of thermal pyrolysis of solvent extracted wax and wax-oil samples collected from the impinger. The units are $\mathrm{mg}$ compound per $\mathrm{g}$ resultant liquid product.

\begin{tabular}{|c|c|c|c|c|c|c|c|}
\hline Compound Name & $\mathbf{M}+$ & Formula & $\begin{array}{l}\text { RT } \\
\text { min }\end{array}$ & $\begin{array}{c}\text { ExtWax } \\
\mathrm{mg} / \mathrm{g}\end{array}$ & $\begin{array}{c}\text { WO450 } \\
\mathrm{mg} / \mathrm{g}\end{array}$ & $\begin{array}{c}\text { WO500 } \\
\mathrm{mg} / \mathrm{g}\end{array}$ & $\begin{array}{c}\text { WO550 } \\
\mathrm{mg} / \mathrm{g}\end{array}$ \\
\hline 1-Hexene & 84 & $\mathrm{C}_{6} \mathrm{H}_{12}$ & 2.1 & - & 237.60 & 201.66 & 117.74 \\
\hline 2-Hexanone, 3,4-dimethyl- & 128 & $\mathrm{C}_{8} \mathrm{H}_{16} \mathrm{O}$ & 2.16 & - & 16.60 & 32.99 & 30.92 \\
\hline Furan, 3-methyl- & 82 & $\mathrm{C}_{5} \mathrm{H}_{6} \mathrm{O}$ & 2.2 & - & 13.40 & 26.74 & 10.43 \\
\hline Cyclopentene, 3-methyl- & 82 & $\mathrm{C}_{6} \mathrm{H}_{10}$ & 2.63 & - & 20.97 & 6.68 & 5.49 \\
\hline Acetic anhydride & 102 & $\mathrm{C}_{4} \mathrm{H}_{6} \mathrm{O}_{3}$ & 2.75 & - & 27.14 & 45.83 & 38.59 \\
\hline Cyclohexene & 82 & $\mathrm{C}_{6} \mathrm{H}_{10}$ & 2.94 & - & 9.66 & 9.36 & 8.62 \\
\hline 1-Heptene & 98 & $\mathrm{C}_{7} \mathrm{H}_{14}$ & 3.06 & 193.37 & 133.04 & 72.83 & 51.18 \\
\hline Hexane, 3-methyl- & 100 & $\mathrm{C}_{7} \mathrm{H}_{16}$ & 3.18 & 12.06 & 16.14 & 19.03 & 20.53 \\
\hline 1,3-Pentadiene, 2,3-dimethyl- & 96 & $\mathrm{C}_{7} \mathrm{H}_{12}$ & 3.24 & 3.08 & 2.77 & 2.83 & 2.62 \\
\hline 2-Heptene & 98 & $\mathrm{C}_{7} \mathrm{H}_{14}$ & 3.28 & 3.32 & 5.21 & 3.15 & 2.11 \\
\hline Cyclopentane, 1-methyl-2-methylene- & 96 & $\mathrm{C}_{7} \mathrm{H}_{12}$ & 3.36 & 1.59 & - & 6.84 & 15.45 \\
\hline 2,3-Dimethyl-1,3-pentadiene & 96 & $\mathrm{C}_{7} \mathrm{H}_{12}$ & 3.4 & 2.66 & - & 3.35 & 1.68 \\
\hline Cyclohexene, 1-methyl- & 96 & $\mathrm{C}_{7} \mathrm{H}_{12}$ & 3.5 & 3.99 & 4.52 & - & 11.08 \\
\hline Cyclohexane, methyl- & 98 & $\mathrm{C}_{7} \mathrm{H}_{14}$ & 3.55 & 3.13 & 5.83 & - & 3.67 \\
\hline Vinylcyclopentane & 96 & $\mathrm{C}_{7} \mathrm{H}_{12}$ & 3.58 & 1.55 & - & - & 2.75 \\
\hline Cyclohexene, 3-methyl- & 96 & $\mathrm{C}_{7} \mathrm{H}_{12}$ & 3.83 & 7.06 & 10.56 & - & 16.30 \\
\hline Methylenecyclohexane & 96 & $\mathrm{C}_{7} \mathrm{H}_{12}$ & 3.88 & 5.18 & 7.81 & 7.96 & 7.22 \\
\hline 1-Undecyne & 152 & $\mathrm{C}_{11} \mathrm{H}_{20}$ & 4.12 & 6.23 & - & - & - \\
\hline Toluene & 92 & $\mathrm{C}_{7} \mathrm{H}_{8}$ & 4.35 & 6.65 & 10.62 & 19.38 & 23.67 \\
\hline 1-Methylcyclohexene & 96 & $\mathrm{C}_{7} \mathrm{H}_{12}$ & 4.39 & 6.55 & 6.77 & - & - \\
\hline 2,4-Hexadiene & 82 & $\mathrm{C}_{6} \mathrm{H}_{10}$ & 4.49 & 3.20 & - & 3.84 & 4.02 \\
\hline 1,4-Octadiene & 110 & $\mathrm{C}_{8} \mathrm{H}_{14}$ & 4.62 & 6.45 & - & 6.78 & 5.94 \\
\hline Heptane, 3-methylene- & 112 & $\mathrm{C}_{8} \mathrm{H}_{16}$ & 4.76 & 66.29 & 89.23 & 57.45 & 54.56 \\
\hline Cyclopropane, pentyl- & 112 & $\mathrm{C}_{8} \mathrm{H}_{16}$ & 4.8 & 165.70 & 114.24 & 6.08 & 6.09 \\
\hline Hexane, 3-ethyl- & 114 & $\mathrm{C}_{8} \mathrm{H}_{18}$ & 4.98 & 12.56 & 6.16 & 5.14 & 7.93 \\
\hline 2-Octene, (Z)- & 112 & $\mathrm{C}_{8} \mathrm{H}_{16}$ & 5.14 & 5.65 & 3.33 & - & - \\
\hline 2-Hexene, 3,5-dimethyl- & 112 & $\mathrm{C}_{8} \mathrm{H}_{16}$ & 5.21 & 2.51 & 0.89 & 2.64 & - \\
\hline
\end{tabular}




\begin{tabular}{|c|c|c|c|c|c|c|c|}
\hline Compound Name & $\mathbf{M}+$ & Formula & $\begin{array}{l}\text { RT } \\
\min \end{array}$ & $\begin{array}{c}\text { ExtWax } \\
\mathrm{mg} / \mathrm{g}\end{array}$ & $\begin{array}{l}\text { WO450 } \\
\mathrm{mg} / \mathrm{g}\end{array}$ & $\begin{array}{l}\text { WO500 } \\
\mathrm{mg} / \mathrm{g}\end{array}$ & $\begin{array}{c}\text { WO550 } \\
\mathrm{mg} / \mathrm{g}\end{array}$ \\
\hline cis-1-Butyl-2-methylcyclopropane & 112 & $\mathrm{C}_{8} \mathrm{H}_{16}$ & 5.32 & 1.99 & 1.11 & - & 3.90 \\
\hline Vinylcyclohexane & 110 & $\mathrm{C}_{8} \mathrm{H}_{14}$ & 5.51 & 2.22 & 3.70 & 1.94 & - \\
\hline 1-Methyl-2-methylenecyclohexane & 110 & $\mathrm{C}_{8} \mathrm{H}_{14}$ & 5.57 & 4.73 & 1.54 & 2.60 & 3.96 \\
\hline 3-Isopropylcyclopentene & 110 & $\mathrm{C}_{8} \mathrm{H}_{14}$ & 6.13 & 0.84 & 0.70 & 8.22 & 4.61 \\
\hline Ethylidenecyclohexane & 110 & $\mathrm{C}_{8} \mathrm{H}_{14}$ & 6.21 & 1.84 & 1.79 & 23.88 & 23.81 \\
\hline 7-Methyl-1-octene & 126 & $\mathrm{C}_{9} \mathrm{H}_{18}$ & 6.33 & 3.15 & 3.41 & 2.84 & 2.36 \\
\hline Ethylbenzene & 106 & $\mathrm{C}_{8} \mathrm{H}_{10}$ & 6.53 & 2.80 & 2.84 & 4.86 & 8.24 \\
\hline p-Xylene & 106 & $\mathrm{C}_{8} \mathrm{H}_{10}$ & 6.73 & 1.82 & 2.82 & 13.39 & 13.18 \\
\hline Cyclohexane, 2-propenyl- & 124 & $\mathrm{C}_{9} \mathrm{H}_{16}$ & 6.91 & 3.81 & 0.87 & - & 2.16 \\
\hline 1,8-Nonadiene & 124 & $\mathrm{C}_{9} \mathrm{H}_{16}$ & 7.05 & 5.09 & 1.16 & 2.03 & 4.90 \\
\hline 1-Nonene & 126 & $\mathrm{C}_{9} \mathrm{H}_{18}$ & 7.28 & 160.02 & 73.67 & 76.82 & 110.92 \\
\hline Nonane & 128 & $\mathrm{C}_{9} \mathrm{H}_{20}$ & 7.51 & 9.43 & 5.95 & 7.88 & 9.17 \\
\hline 2-Nonene, (E)- & 128 & $\mathrm{C}_{9} \mathrm{H}_{20}$ & 7.69 & 1.57 & 1.91 & 1.67 & 1.37 \\
\hline $\begin{array}{l}\text { Cyclopentane, 1-methyl-2-(2-propenyl)-, } \\
\text { trans- }\end{array}$ & 124 & $\mathrm{C}_{9} \mathrm{H}_{16}$ & 7.8 & 2.35 & 1.21 & 9.07 & 9.88 \\
\hline cis-2-Nonene & 126 & $\mathrm{C}_{9} \mathrm{H}_{18}$ & 7.92 & 0.70 & 3.36 & 3.13 & 2.60 \\
\hline Allylcyclohexane & 124 & $\mathrm{C}_{9} \mathrm{H}_{16}$ & 8.11 & 1.95 & 1.01 & 1.94 & 3.06 \\
\hline 1-Butylcyclopentene & 124 & $\mathrm{C}_{9} \mathrm{H}_{16}$ & 8.9 & 1.70 & 1.48 & 1.99 & 2.20 \\
\hline 4-Dodecene, (E)- & 168 & $\mathrm{C}_{12} \mathrm{H}_{24}$ & 9.06 & 0.89 & 1.14 & 2.02 & 3.08 \\
\hline 4-Nonene, 2-methyl- & 140 & $\mathrm{C}_{10} \mathrm{H}_{20}$ & 9.13 & 2.41 & 0.96 & 3.14 & 2.86 \\
\hline 1-Methyl-2-(3-methylpentyl) cyclopropane & 140 & $\mathrm{C}_{10} \mathrm{H}_{20}$ & 9.31 & 1.77 & 1.31 & 3.56 & 5.47 \\
\hline 1,11-Dodecadiene & 166 & $\mathrm{C}_{12} \mathrm{H}_{22}$ & 9.76 & 1.27 & 1.01 & 7.13 & 4.67 \\
\hline Bicyclo[3.3.1]nonan-2-one & 138 & $\mathrm{C}_{9} \mathrm{H}_{14} \mathrm{O}$ & 9.93 & - & - & - & 15.55 \\
\hline 1-Decene & 140 & $\mathrm{C}_{10} \mathrm{H}_{20}$ & 10.18 & 130.92 & 71.77 & 89.19 & 141.42 \\
\hline Mesitylene & 120 & $\mathrm{C}_{9} \mathrm{H}_{12}$ & 10.27 & - & - & 2.99 & - \\
\hline Decane & 140 & $\mathrm{C}_{10} \mathrm{H}_{20}$ & 10.43 & 6.05 & 5.06 & 8.83 & 8.84 \\
\hline 4-Decene & 140 & $\mathrm{C}_{10} \mathrm{H}_{20}$ & 10.6 & 1.47 & 0.22 & 2.68 & 3.02 \\
\hline Cyclohexane, 1-methyl-2-propyl- & 140 & $\mathrm{C}_{10} \mathrm{H}_{20}$ & 10.86 & 1.34 & 0.72 & 0.83 & 1.48 \\
\hline Bicyclo[6.1.0]nonane & 124 & $\mathrm{C}_{9} \mathrm{H}_{16}$ & 11.14 & 1.01 & 0.56 & 2.18 & 2.04 \\
\hline Cyclotene & 112 & $\mathrm{C}_{6} \mathrm{H}_{8} \mathrm{O}_{2}$ & 11.27 & - & - & - & 3.87 \\
\hline 2-Cyclopenten-1-one, 2,3-dimethyl- & 110 & $\mathrm{C}_{7} \mathrm{H}_{10} \mathrm{O}$ & 11.67 & - & - & - & 1.60 \\
\hline 1,10-Undecadiene & 152 & $\mathrm{C}_{11} \mathrm{H}_{20}$ & 12.09 & 0.89 & 0.58 & 3.59 & 1.18 \\
\hline Phenol, 2-methyl- & 108 & $\mathrm{C}_{7} \mathrm{H}_{8} \mathrm{O}$ & 12.13 & - & - & - & 4.36 \\
\hline p-Cresol & 108 & $\mathrm{C}_{7} \mathrm{H}_{8} \mathrm{O}$ & 12.75 & - & - & - & 4.77 \\
\hline 1-Dodecene & 168 & $\mathrm{C}_{12} \mathrm{H}_{24}$ & 13.17 & 60.01 & 36.77 & 70.82 & 76.11 \\
\hline Tetradecane & 198 & $\mathrm{C}_{14} \mathrm{H}_{30}$ & 13.41 & 2.90 & 3.24 & 6.66 & 3.20 \\
\hline Nonanal & 142 & $\mathrm{C}_{9} \mathrm{H}_{18} \mathrm{O}$ & 13.57 & - & 1.76 & 2.01 & 1.77 \\
\hline 2-Cyclopenten-1-one, 3-ethyl-2-hydroxy- & 126 & $\mathrm{C}_{7} \mathrm{H}_{10} \mathrm{O}_{2}$ & 14.06 & - & - & - & 0.40 \\
\hline
\end{tabular}




\begin{tabular}{|l|c|c|c|c|c|c|c|}
\hline Compound Name & $\mathbf{M}+$ & Formula & $\begin{array}{c}\text { RT } \\
\mathbf{m i n}\end{array}$ & $\begin{array}{c}\text { ExtWax } \\
\mathbf{m g} / \mathbf{g}\end{array}$ & $\begin{array}{c}\text { WO450 } \\
\mathbf{m g} / \mathbf{g}\end{array}$ & $\begin{array}{c}\text { WO500 } \\
\mathbf{m g} / \mathbf{g}\end{array}$ & $\begin{array}{c}\text { WO550 } \\
\mathbf{m g} / \mathbf{g}\end{array}$ \\
\hline Benzene, (1-methylene-2-propenyl)- & 130 & $\mathrm{C}_{10} \mathrm{H}_{10}$ & 14.93 & - & - & - & 2.92 \\
\hline Dodecane & 170 & $\mathrm{C}_{12} \mathrm{H}_{26}$ & 16.09 & 23.35 & 11.86 & 43.44 & 25.33 \\
\hline 3-Dodecene, (Z)- & 168 & $\mathrm{C}_{12} \mathrm{H}_{24}$ & 16.23 & - & 0.93 & 2.57 & 1.02 \\
\hline Phenol, 4-ethyl-2-methoxy- & 152 & $\mathrm{C}_{9} \mathrm{H}_{12} \mathrm{O} 2$ & 18.66 & - & 1.58 & 1.34 & 1.04 \\
\hline 1-Tridecene & 182 & $\mathrm{C}_{13} \mathrm{H}_{26}$ & 18.89 & 11.53 & 3.51 & 21.33 & 17.52 \\
\hline 2-n-Propyl-1-heptanol & 158 & $\mathrm{C}_{10} \mathrm{H}_{22} \mathrm{O}$ & 19.09 & - & 0.31 & 1.46 & 0.60 \\
\hline 1-Tetradecene & 196 & $\mathrm{C}_{14} \mathrm{H}_{28}$ & 21.55 & 9.61 & 1.44 & 10.37 & 4.84 \\
\hline 1-Pentadecene & 210 & $\mathrm{C}_{15} \mathrm{H}_{30}$ & 24.05 & 8.16 & 0.67 & 0.67 & 2.81 \\
\hline 1-Hexadecene & 224 & $\mathrm{C}_{16} \mathrm{H}_{32}$ & 26.43 & 6.05 & 0.34 & 5.83 & 1.71 \\
\hline 1-Heptadecene & 238 & $\mathrm{C}_{17} \mathrm{H}_{34}$ & 28.69 & 3.81 & 3.22 & 3.02 & 1.17 \\
\hline 1-Octadecene & 252 & $\mathrm{C}_{18} \mathrm{H}_{36}$ & 30.83 & 1.75 & - & 1.52 & 0.42 \\
\hline
\end{tabular}


Table S5. Carbon number partitioning of alkanes, olefins, aromatics, and oxygenated compounds in the non-catalytic and catalytic pyrolysis products of solvent extracted wax and wax-oil samples (WO450, WO500, WO550). The proportions are also shown for gasoline as a basis of comparison. Units are number of carbons.

\begin{tabular}{|c|c|c|c|c|c|c|c|c|c|c|c|c|c|}
\hline \multirow{2}{*}{ Pyrolysis } & \multirow{2}{*}{$\begin{array}{c}\text { Sample } \\
\text { Name }\end{array}$} & \multicolumn{3}{|c|}{ Alkanes } & \multicolumn{3}{|c|}{ Olefins } & \multicolumn{3}{|c|}{ Aromatics } & \multicolumn{3}{|c|}{ Oxygenated } \\
\hline & & Max & Min & Range & Max & Min & Range & Max & Min & Range & Max & Min & Range \\
\hline \multirow{4}{*}{ 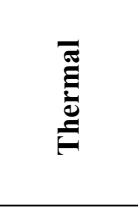 } & Ext Wax & 31 & 7 & 25 & 31 & 6 & 26 & 8 & 0 & 1 & 17 & 0 & 1 \\
\hline & WO0450 & 39 & 7 & 33 & 34 & 6 & 29 & 8 & 7 & 2 & 17 & 3 & 2 \\
\hline & WO500 & 39 & 7 & 33 & 34 & 6 & 29 & 9 & 7 & 3 & 12 & 3 & 3 \\
\hline & WO550 & 38 & 7 & 32 & 34 & 6 & 29 & 10 & 7 & 4 & 15 & 3 & 4 \\
\hline \multirow{4}{*}{ 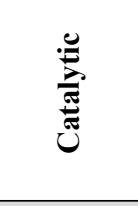 } & Ext Wax & 15 & 6 & 10 & 10 & 6 & 5 & 13 & 7 & 7 & & & \\
\hline & WO450 & 15 & 6 & 10 & 10 & 6 & 5 & 13 & 7 & 7 & & & \\
\hline & WO500 & 15 & 6 & 10 & 10 & 6 & 5 & 13 & 7 & 7 & & & \\
\hline & WO550 & 15 & 6 & 10 & 10 & 6 & 5 & 13 & 7 & 7 & & & \\
\hline \multicolumn{2}{|c|}{ Gasoline } & 13 & 6 & 8 & 10 & 6 & 5 & 12 & 7 & 6 & & & \\
\hline
\end{tabular}


Table S6. Carbon amount ( $\mathrm{g} \mathrm{C/} 100 \mathrm{~g}$ resultant liquid) in alkanes, olefins, aromatics, and oxygenated compounds in the pyrolysis thermal and catalytic) products from solvent extracted wax and wax-oil samples (WO450, WO500, WO550). The proportions are also shown for gasoline as a basis of comparison.

\begin{tabular}{|c|c|c|c|c|c|c|}
\hline \multirow{2}{*}{ Pyrolysis } & \multirow{2}{*}{$\begin{array}{l}\text { Sample } \\
\text { Name }\end{array}$} & \multicolumn{2}{|c|}{ Alkanes (g/100 g) } & \multirow{2}{*}{$\begin{array}{l}\text { Olefins } \\
\text { (g/100 g) }\end{array}$} & \multirow{2}{*}{$\begin{array}{c}\text { Aromatics } \\
(\mathrm{g} / \mathbf{1 0 0} \mathrm{g})\end{array}$} & \multirow{2}{*}{$\begin{array}{c}\text { Oxygenated } \\
\text { (g/100 g) }\end{array}$} \\
\hline & & Branched & Straight & & & \\
\hline \multirow{4}{*}{ 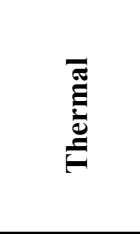 } & ExtWax & 9.53 & 39.19 & 46.57 & - & 4.61 \\
\hline & WO450 & 6.81 & 56.01 & 32.47 & 0.31 & 4.41 \\
\hline & WO500 & 11.15 & 57.12 & 28.57 & 0.43 & 2.73 \\
\hline & WO550 & 9.68 & 45.38 & 40.06 & 0.35 & 4.54 \\
\hline \multirow{4}{*}{ Ũ } & ExtWax & 50.89 & 1.64 & 7.17 & 40.60 & - \\
\hline & WO450 & 46.64 & 1.51 & 5.03 & 46.82 & - \\
\hline & WO500 & 41.66 & 2.16 & 11.72 & 44.35 & - \\
\hline & WO550 & 53.37 & 1.09 & 7.31 & 38.23 & - \\
\hline \multicolumn{2}{|c|}{ Gasoline } & 46.9 & 0.5 & 4.00 & 48.6 & - \\
\hline
\end{tabular}




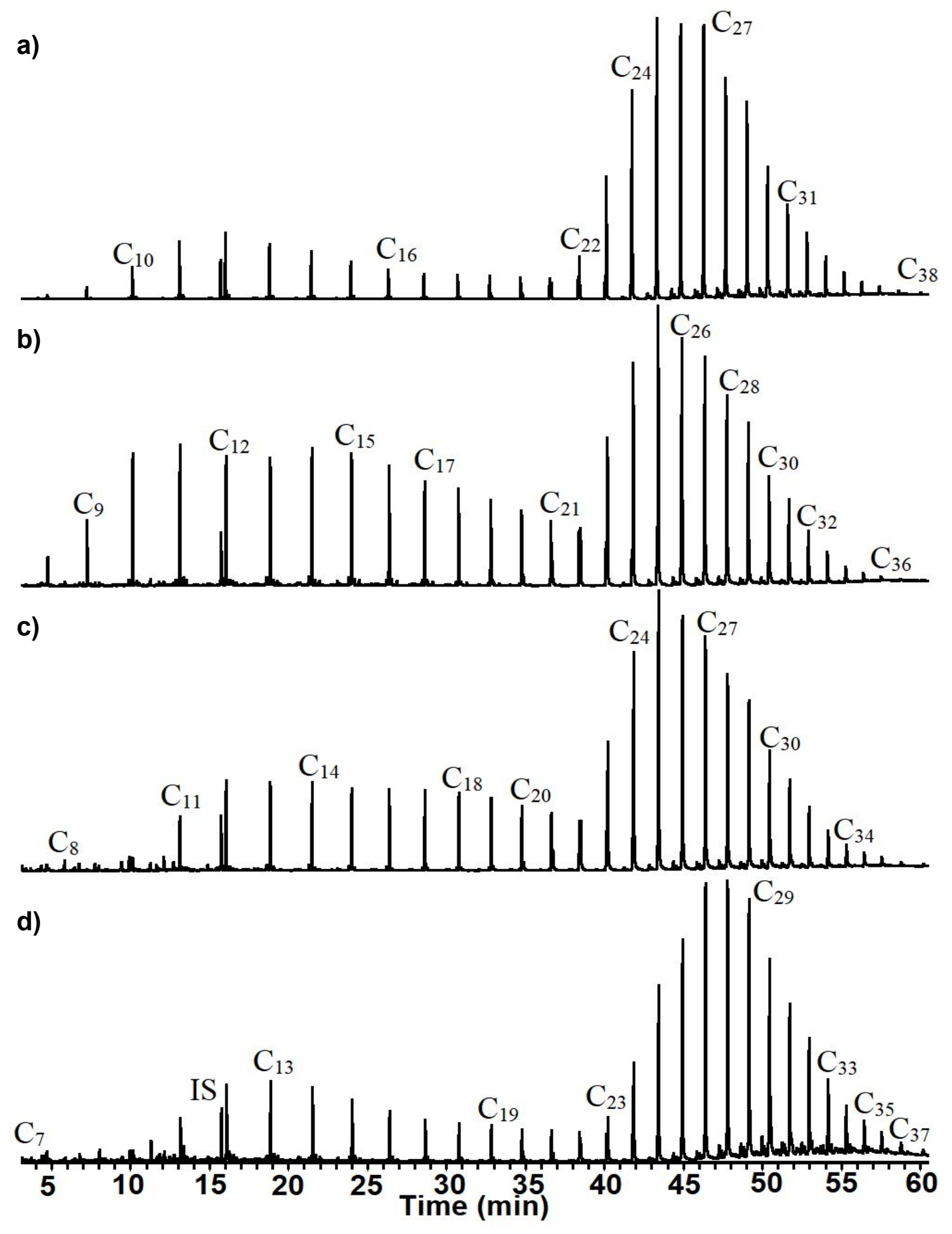

Figure S1. GC-MS Chromatograms of thermal pyrolysis products of (a) solvent extracted waxpy, (b) WO450-py, (c) WO500-py, and (d) WO550-py collected from the U-tube. 


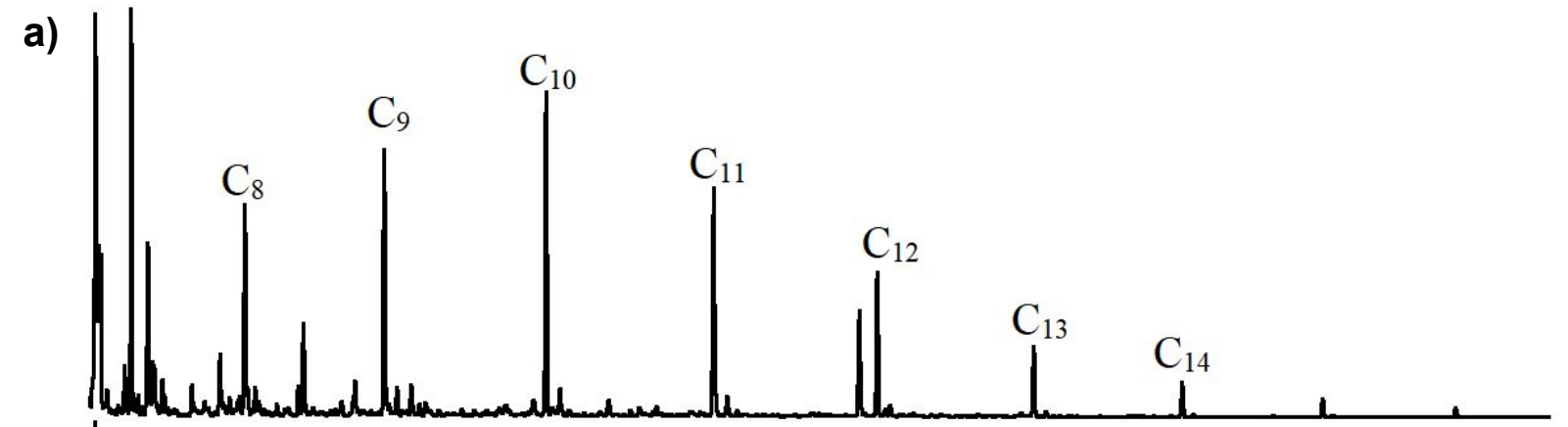

b)

c)
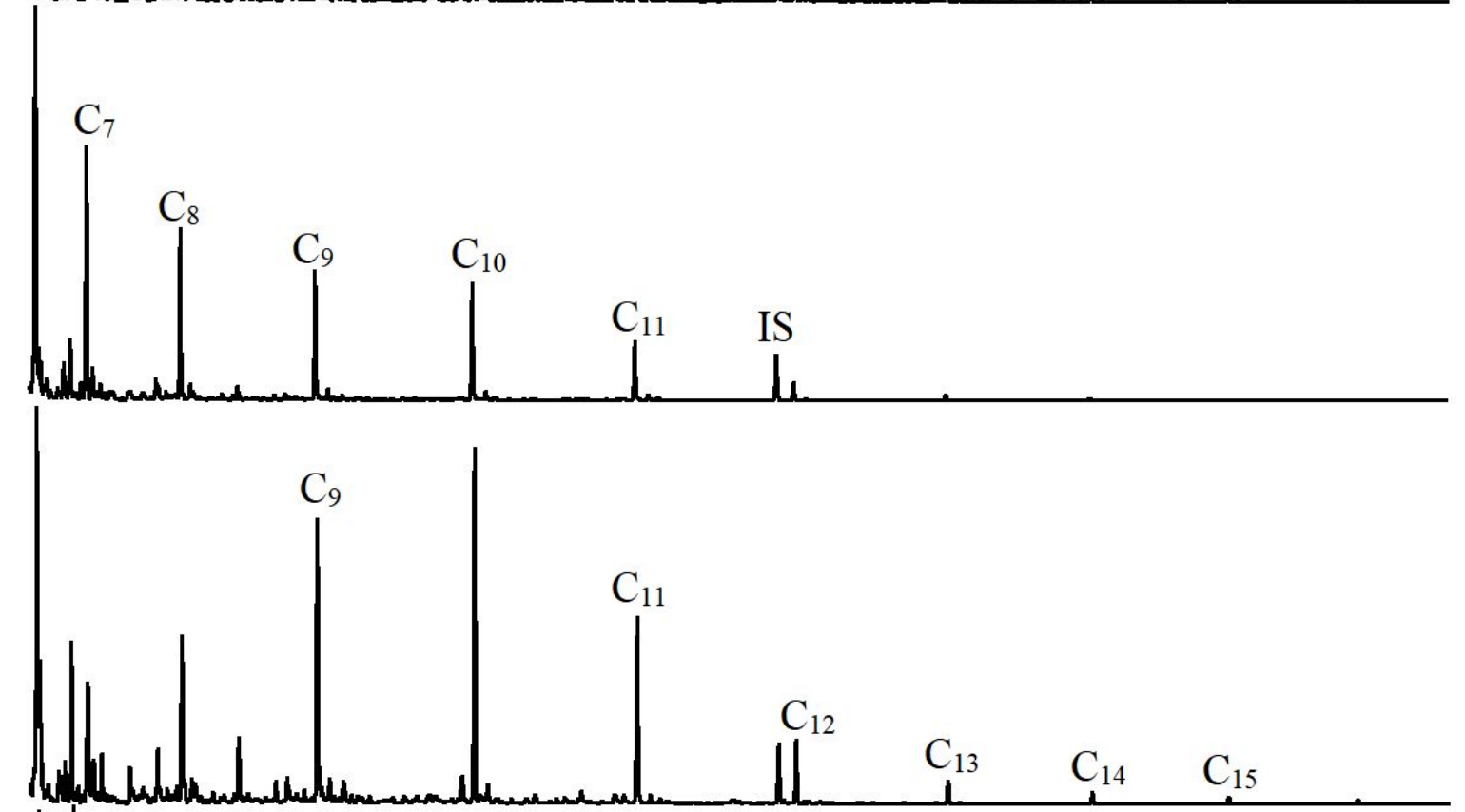

d)

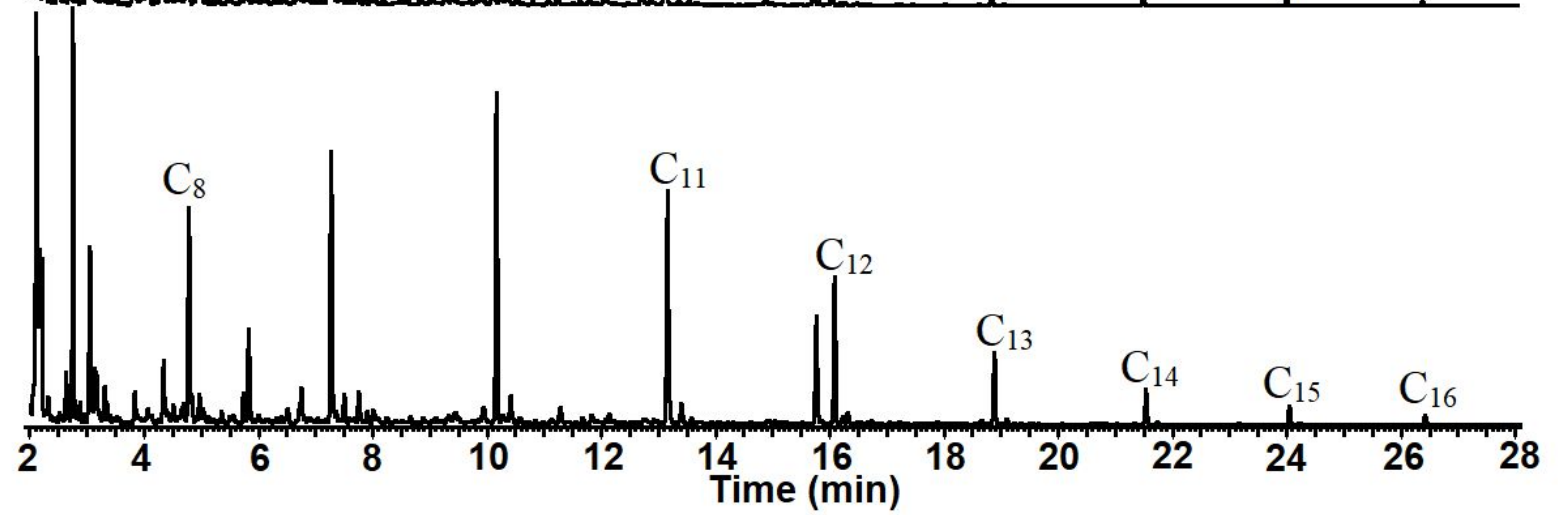

Figure S2. GC-MS Chromatograms of thermal pyrolysis products of (a) solvent extracted waxpy, (b) WO450-py, (c) WO500-py, and (d) WO550-py collected from the impinger. 


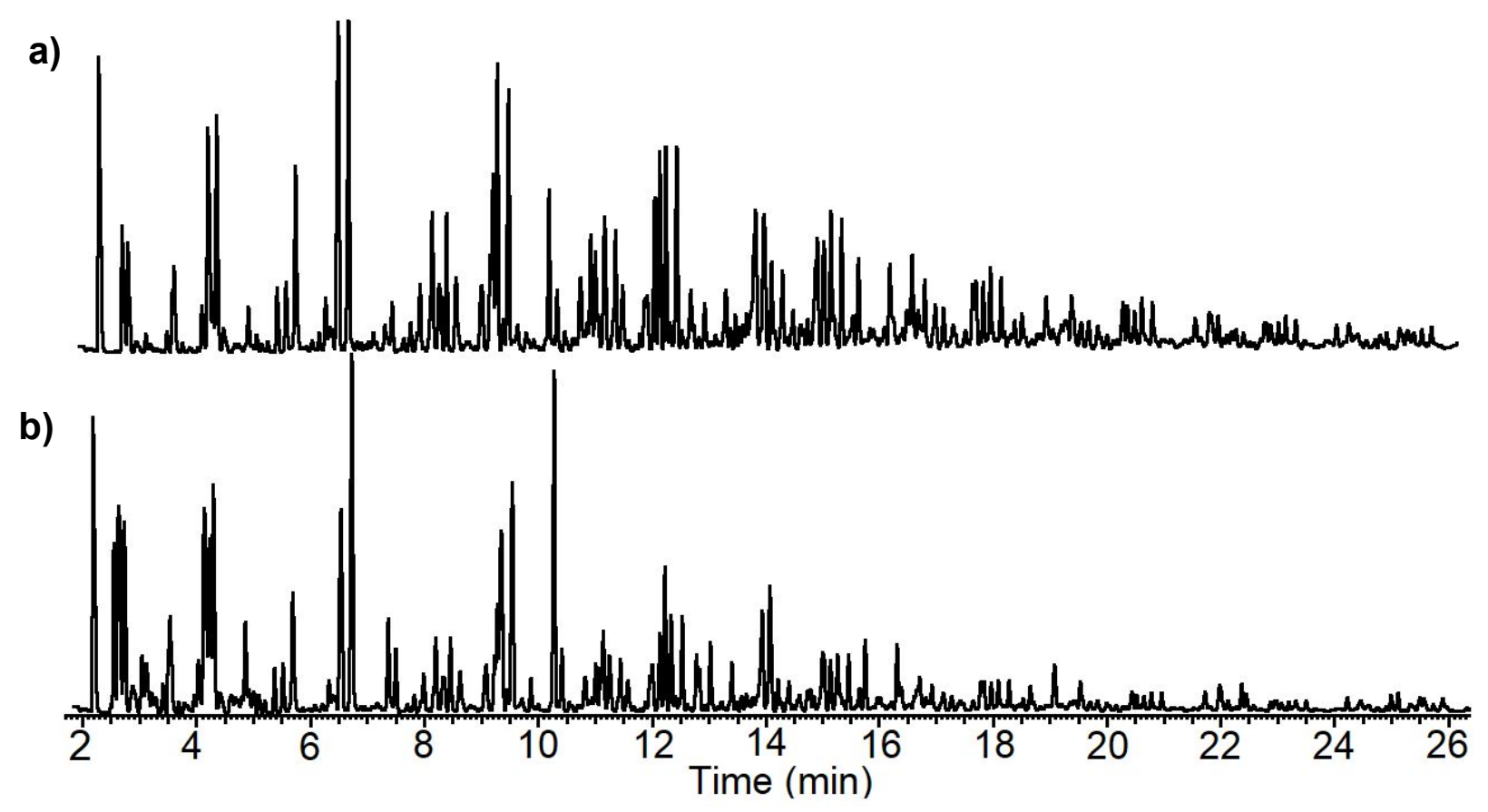

Figure S3. GC-MS Chromatograms of catalytic pyrolysis products from the U-tube condenser of (a) solvent extracted wax-cat virgin catalyst and (b) solvent extracted wax-cat $5^{\text {th }}$ time reused catalyst.

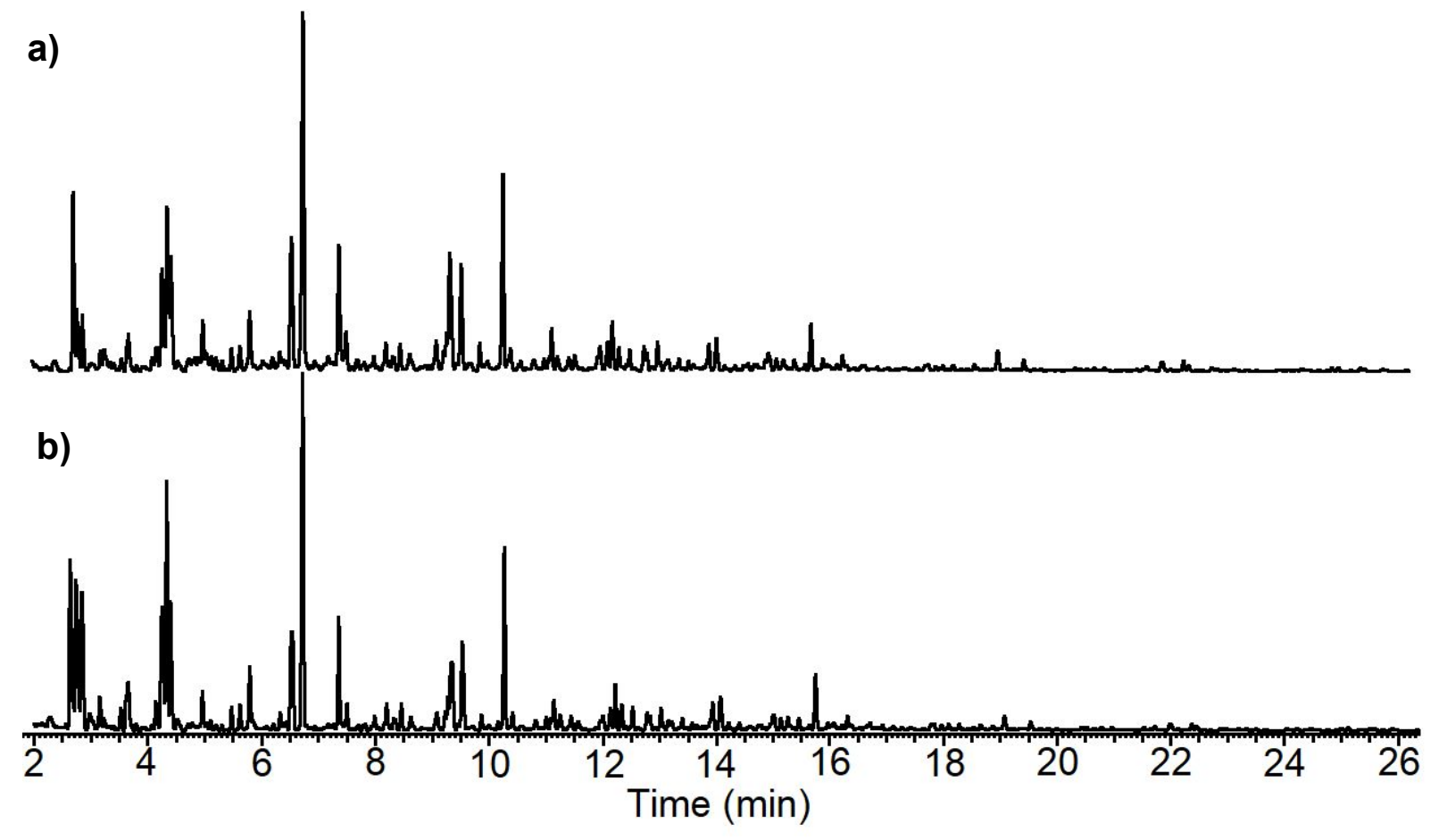

Figure S4. GC-MS Chromatograms of catalytic pyrolysis products from the U-tube condenser of (a) WO450-cat virgin catalyst and (b) WO450-cat $5^{\text {th }}$ time reused catalyst. 


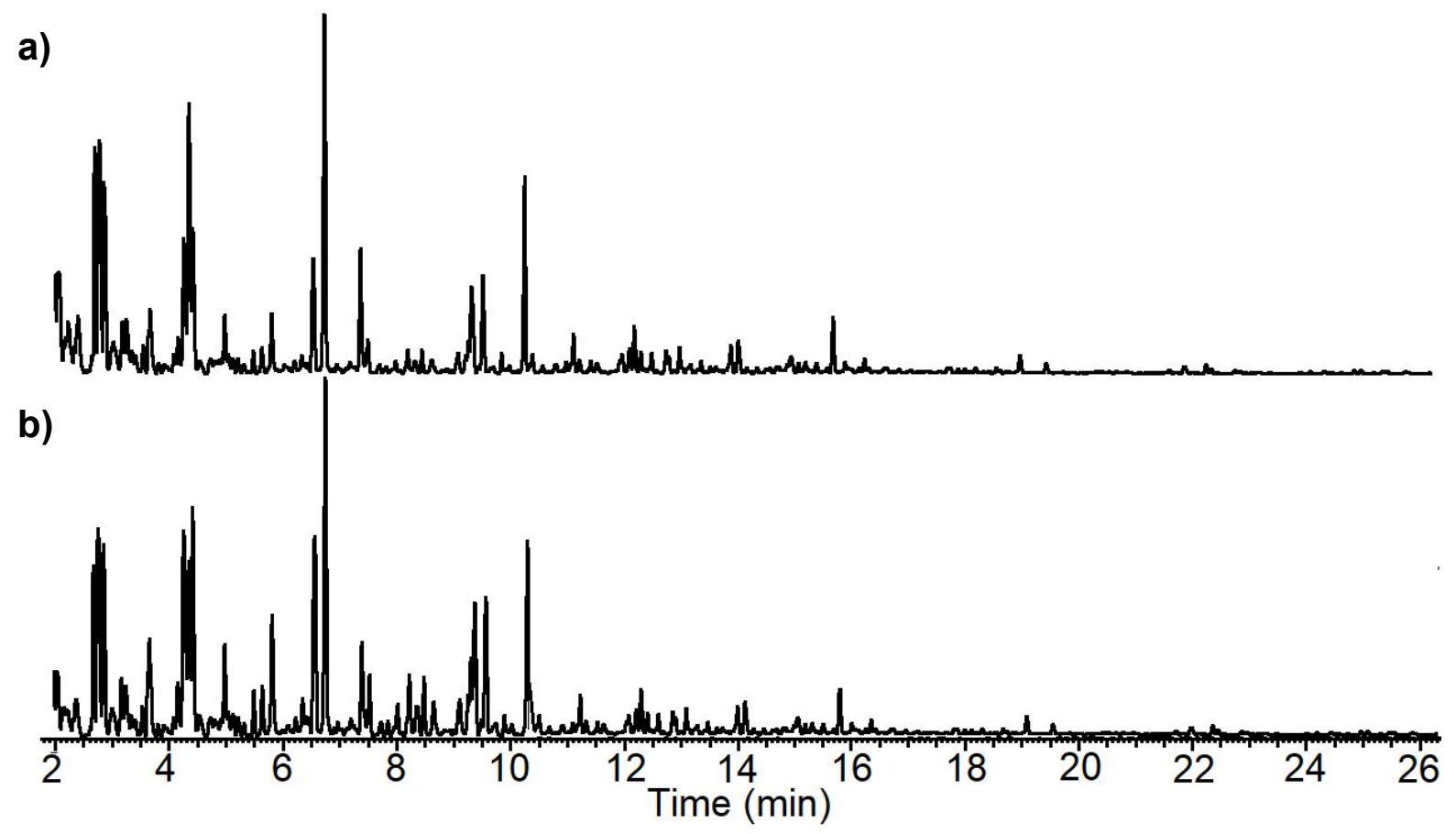

Figure S5. GC-MS Chromatograms of catalytic pyrolysis products from the U-tube condenser of (a) WO500-cat virgin catalyst and (b) WO500-cat $5^{\text {th }}$ time reused catalyst.

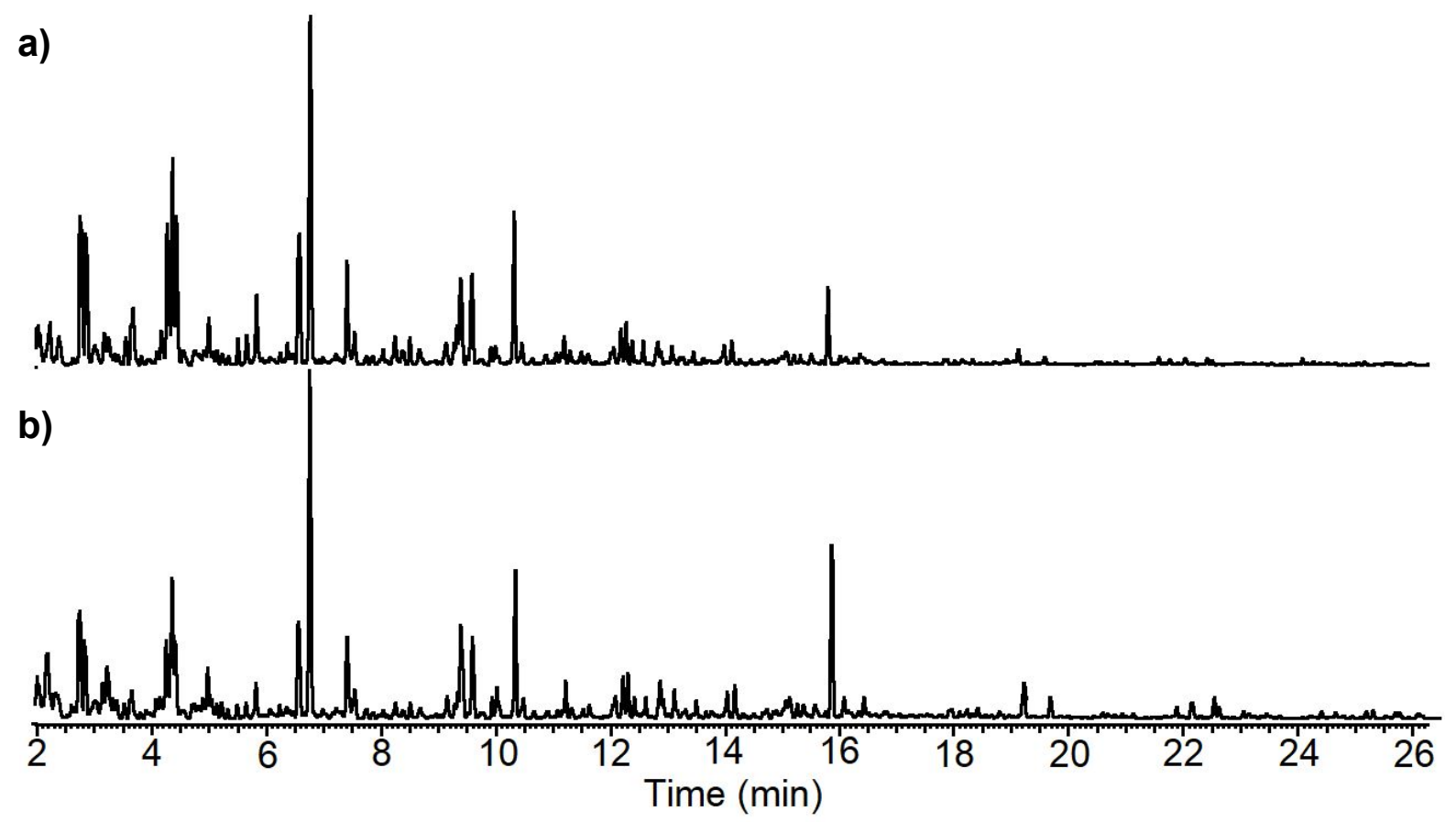

Figure S6. GC-MS Chromatograms of catalytic pyrolysis products from the U-tube condenser of (a) WO550-cat virgin catalyst and (b) WO550-cat $5^{\text {th }}$ time reused catalyst. 